\title{
Extremely metal-poor star-forming galaxies ${ }^{\star}$
}

\section{New detections and general morphological and photometric properties}

\author{
P. Papaderos ${ }^{1,2}$, N. G. Guseva ${ }^{3}$, Y. I. Izotov ${ }^{3}$, and K. J. Fricke ${ }^{4}$ \\ 1 Instituto de Astrofísica de Andalucía (CSIC), Camino Bajo de Huétor 50, Granada 18008, Spain \\ e-mail: papaderos@iaa.es \\ 2 Max-Planck-Institute for Radioastronomy, Auf dem Hügel 69, 53121 Bonn, Germany \\ 3 Main Astronomical Observatory, Ukrainian National Academy of Sciences, 27 Zabolotnoho str., Kyiv 03680, Ukraine \\ ${ }^{4}$ Institute for Astrophysics, University of Göttingen, Friedrich-Hund-Platz 1, 37077 Göttingen, Germany
}

Received 22 April 2008 / Accepted 29 July 2008

\section{ABSTRACT}

\begin{abstract}
Context. Extremely metal-deficient $[12+\log (\mathrm{O} / \mathrm{H}) \lesssim 7.6]$ emission-line galaxies in the nearby universe are invaluable laboratories of extragalactic astronomy and observational cosmology since they allow us to study collective star formation and the evolution of galaxies under chemical conditions approaching those in distant protogalactic systems. However, despite intensive searches over the last three decades, nearby star-forming (SF) galaxies with strongly subsolar metallicity remain extremely scarce.

Aims. We searched the Sloan Digital Sky Survey (SDSS) and the Six-Degree Field Galaxy Redshift Survey (6dFGRS) for promising low-metallicity candidates using a variety of spectroscopic criteria.

Methods. We present long-slit spectroscopy with the $3.6 \mathrm{~m}$ ESO telescope of eight HII regions in seven emission-line dwarf galaxies, selected from the Data Release 4 of SDSS (six galaxies) and from 6dFGRS (one galaxy). In addition, we use SDSS imaging data to investigate the photometric structure of the sample galaxies.

Results. From the $3.6 \mathrm{~m}$ telescope spectra, we determine the oxygen abundance of these systems to be $7.3 \lessgtr 12+\log (\mathrm{O} / \mathrm{H}) \lessgtr 7.6$, placing them among the most metal-poor star-forming galaxies ever discovered. Our photometric analysis reveals a moderately blue, stellar host galaxy in all sample galaxies.

Conclusions. The detection of a stellar host in all galaxies studied here and all previously studied extremely metal-deficient SF galaxies implies that they are unlikely to be forming their first generation of stars. With regard to the structural properties of their host galaxy, we demonstrate that these systems are indistinguishable from blue compact dwarf (BCD) galaxies. However, in contrast to the majority ( $>90 \%$ ) of BCDs that are characterised by red elliptical host galaxies, extremely metal-poor SF dwarfs (hereafter XBCDs) reveal moderately blue and irregular hosts. This is consistent with a young evolutionary status and in the framework of standard star formation histories implies that several XBCDs formed most of their stellar mass in the past $\sim 2$ Gyr. A large fraction of XBCDs reveal a cometary morphology due to the presence of intense SF activity at one edge of an elongated host galaxy with a gradually decreasing surface brightness towards its antipodal end.
\end{abstract}

Key words. galaxies: dwarf - galaxies: starburst - galaxies: abundances - galaxies: structure - galaxies: evolution

\section{Introduction}

The identification and detailed studies of chemically unevolved, star-forming (SF) galaxies in the nearby universe, of almost pristine chemical composition, is a major task for contemporary observational cosmology. Some important aspects of these studies are the following.

First, systematic studies of SF galaxies with strongly subsolar metal abundances are indispensable for placing tight observational constraints on the primordial ${ }^{4} \mathrm{He}$ abundance $Y_{\mathrm{p}}$ (Izotov et al. 2007a; Peimbert et al. 2007, and references therein).

Secondly, the metallicity plays a key role in virtually all aspects of star - and galaxy evolution since it influences e.g. a) the production rate of Lyman continuum photons and efficiency of radiative winds in massive stars (see e.g. Leitherer et al. 1992; Schaerer \& de Koter 1997; Kudritzki 2002), including the properties of Wolf-Rayet stellar populations

* Based on observations collected at the European Southern Observatory, Chile, ESO programs 075.B-0768 and 076.B-0739.
(Izotov et al. 1997a; Schaerer \& Vacca 1998; Guseva et al. 2000; Crowther \& Hadfield 2006; Brinchman et al. 2008), as well as b) the cooling efficiency of the hot $\left(\sim 10^{7} \mathrm{~K}\right)$ X-ray emitting gas (Böhringer \& Hensler 1989), recognised to be an ubiquitous component of starburst galaxies (see e.g. Heckman et al. 1995; Papaderos \& Fricke 1998; Martin et al. 2002; Ott et al. 2005). Spatially resolved studies of extremely metal-poor nearby SF galaxies may therefore yield crucial insights into the early evolution of faint protogalactic systems forming out of primordial or almost metal-free gas in the young universe.

The most suitable nearby objects for exploring these issues are blue compact dwarf (BCD) galaxies. These galaxies form a morphologically heterogeneous class of intrinsically faint $\left(M_{B} \gtrsim\right.$ $-18 \mathrm{mag}$ ) extragalactic systems undergoing intense starburst activity on a spatial scale of typically $\sim 1 \mathrm{kpc}$ (see Papaderos et al. 2002 , hereafter P02 and references therein). In $\sim 90 \%$ of the local BCD population, SF activity proceeds in one or several luminous HII regions within the central part of a more extended, low-surface brightness (LSB) elliptical host galaxy (Loose \& Thuan 1986; Papaderos et al. 1996a, hereafter LT86 and P96a, 
respectively). This red $(0.8 \lesssim B-R$ (mag) $\lesssim 1.2$; P96a) galaxy host contains, on average, one half of the optical emission of a BCD (Papaderos et al. 1996b, hereafter P96b) and typically dominates the line-of-sight intensity of this system for surface brightness levels fainter than $24.5 \mathrm{~B}$ mag/ $\square^{\prime \prime}$ (P96a, P02). Deep surface photometry in the optical and near infrared (Noeske et al. 2003; Cairós et al. 2003; Gil de Paz \& Madore 2005) and colour-magnitude-diagram analyses (e.g., Schulte-Ladbeck et al. 1999; Tosi et al. 2001) confirmed the earlier conclusion (LT86, P96b) that BCDs are, overwhelmingly, evolved gas-rich dwarfs undergoing recurrent starburst activity.

BCDs are the most metal-deficient emission-line galaxies known in the nearby universe. However, while all BCDs show subsolar chemical abundances, it is notoriously difficult to find extremely metal-deficient systems in the range $12+\log (\mathrm{O} / \mathrm{H}) \lesssim$ 7.6. The BCD abundance distribution peaks at $12+\log (\mathrm{O} / \mathrm{H}) \approx$ 8.1 with a sharp drop-off at lower values (Terlevich et al. 1991; Thuan et al. 1995; Izotov \& Thuan 1998a; Kunth \& Östlin 2000). One of the first BCDs discovered, IZw 18 (Sargent \& Searle 1970) with $12+\log (\mathrm{O} / \mathrm{H})=7.17 \pm 0.01$ (Izotov et al. 1997a), held the record as the most metal-deficient SF galaxy known for more than three decades. Only very recently was this system replaced in the metallicity ranking by the BCD SBS 0335-052 W with an oxygen abundance $12+\log (\mathrm{O} / \mathrm{H})=7.12 \pm 0.03$ (Izotov et al. 2005).

Despite large observational efforts over the past three decades and systematic studies of several $10^{5}$ catalogued emission-line galaxies, less than 20 BCDs with $12+\log (\mathrm{O} / \mathrm{H}) \lessgtr$ 7.6 (hereafter XBCDs) were identified in the nearby $(z \lesssim 0.04)$ universe until a few years ago (Kunth \& Östlin 2000, and references therein). Since then substantial progress has been achieved, and more than one dozen further XBCDs discovered (Kniazev et al. 2003, 2004; Guseva et al. 2003a,b; Pustilnik et al. 2005; Pisano et al. 2005; Pustilnik et al. 2006; Izotov et al. 2006b; Papaderos et al. 2006b; Izotov \& Thuan 2007; Kewley et al. 2007). At higher redshift $(0.2 \lesssim z \lesssim 0.8)$, dedicated surveys utilising $10 \mathrm{~m}$-class telescopes and optimised search strategies led to the discovery of about ten more SF dwarf galaxies with $12+\log (\mathrm{O} / \mathrm{H}) \lesssim 7.6$ and an absolute rest-frame magnitude in the range of BCDs (Kakazu et al. 2007). In spite of the extreme scarcity and intrinsic faintness of XBCDs, there is therefore a tangible prospect of unveiling a significant number of these systems in the years to come.

Third, recent work provides strong observational support to the idea that some XBCDs in the nearby universe formed most of their stellar mass in the past $\sim 1 \mathrm{Gyr}$, which implies that they are cosmologically young systems (Papaderos et al. 1998; Vanzi et al. 2000; Guseva et al. 2001; Papaderos et al. 2002; Guseva et al. 2003a,b,c; Hunt et al. 2003; Pustilnik et al. 2004a). The youth hypothesis for XBCDs is further supported by a number of evolutionary synthesis and colour-magnitude diagram (CMD) studies that indicate an upper age of 0.1-2 Gyr for the stellar component of some of those systems (Izotov et al. 1997b; Thuan et al. 1997; Izotov et al. 2001; Fricke et al. 2001; Izotov \& Thuan 2004b,c).

In the case of the XBCD I Zw 18, the conclusion that this system started forming stars not earlier than $\lesssim 0.5 \mathrm{Gyr}$ ago (Izotov \& Thuan 2004b), was recently disputed by the subsequent CMD studies of Aloisi et al. (2007).

However, apart from the question of when the first stars in a XBCD were formed, there appears to be broad consensus that many of these systems underwent the dominant phase of their formation only recently. If so, XBCDs may be regarded as convenient laboratories to explore the main processes driving dwarf galaxy formation, as long as their morphological and dynamical relics have not had time to be erased in the course of the secular galactic evolution, through e.g. two-body relaxation, galaxy merging and subsequent star formation episodes. Low-mass protogalaxies in the distant universe, once detected, will remain due to the cosmological dimming and their intrinsic faintness and compactness challenging to study with sufficient resolution and accuracy, even with the next generation of extremely large telescopes. Young XBCD candidates provide in this respect a bridge between near-field and high-redshift observational cosmology and invaluable laboratories of extragalactic research.

This paper investigates the spectroscopic, photometric, and morphological properties of a new sample of XBCDs. It is organised as follows: the sample selection, data acquisition, and reduction are described in Sect. 2. Our spectroscopic and photometric analysis is presented in Sects. 3 and 4, we discuss the properties of individual sample galaxies. The photometric and morphological properties of XBCDs are reviewed in Sects. 5 and 6, we summarise our conclusions.

\section{Sample and observations}

\subsection{Sample selection}

With the aim of finding new XBCD candidates, we carried out a systematic search for these objects in the SDSS Data Release 4 (Adelman-McCarthy et al. 2006), on the basis of the relative fluxes of emission lines. All extremely metal-deficient emissionline galaxies known are characterised by relatively weak (compared to $\mathrm{H} \beta$ ) [O II] $\lambda 3727$, [O III] $\lambda 4959, \lambda 5007$ and [N II] $\lambda 6583$ emission lines (e.g. Izotov \& Thuan 1998a,b; Thuan \& Izotov 2005; Pustilnik et al. 2005). These spectral properties identify uniquely low-metallicity dwarfs, since no other type of galaxy possess these spectral characteristics. We considered additionally spectra in which the [O III] $\lambda 4363$ emission line is weak or not detected. Since the [O II] $\lambda 3727$ line is outside of the observed wavelength range of the SDSS spectra for all galaxies of redshift $z<0.02$, we used the [O III] $\lambda 4959, \lambda 5007$, and [N II] $\lambda 6583$ emission to identify extremely low-metallicity galaxy candidates. Following this strategy (Izotov et al. 2006b), we selected only galaxies with weak [O III] and [N II] emission lines.

We also searched for XBCD candidates in the Six-Degree Field Galaxy Redshift Survey (6dFGRS) (Jones et al. 2005). Our strategy (see also Papaderos et al. 2006b, hereafter P06) was also based on the visual selection of spectra with blue continua and strong emission lines, and for which the [O III] $\lambda 4363$ emission line was present. This is because the [O III] $\lambda 4363$ auroral line is easily detectable in high-temperature and hence lowmetallicity objects. This criterion also selects high-metallicity AGNs, which we rejected by visual examination of each spectrum based on the relative intensities of some emission lines (e.g. strong [O II] $\lambda 3727$ and [N II] $\lambda 6583$ emission in combination with strong [O III] $\lambda 5007$ emission, strong He II $\lambda 4686$, and $[\mathrm{O} \mathrm{I}] \lambda 6300$, etc.).

We describe observations of seven XBCD candidates with the $3.6 \mathrm{~m}$ ESO telescope. Three candidates were reported to be extremely metal-poor galaxies by Kniazev et al. (2003, 2004) on the basis of oxygen abundances obtained from SDSS spectral measurements. However, given that in the SDSS spectra of these three systems, the [O II] $\lambda 3727$ line is out of the range, the value of $\mathrm{O}^{+} / \mathrm{H}^{+}$was calculated from the $[\mathrm{O}$ II] $\lambda 7320,7330$ lines. But since the intensities of these infrared lines are many times lower than that of the [O II] $\lambda 3727$ doublet, this method is 
Table 1. Coordinates of the sample galaxies (J2000.0).

\begin{tabular}{lccccccc}
\hline \hline Name & RA & Dec & Redshift & Distance $^{a}(\mathrm{Mpc})$ & airmass & PA $\left(^{\circ}\right)$ & Exp. time $(\mathrm{s})$ \\
\hline J 0133+1342 & 013352.6 & +134209 & 0.00879 & 37.8 & 1.37 & 170.4 & $3600(3 \times 1200)$ \\
g0405204-364859 & 040520.4 & -364859 & 0.00268 & 11.4 & 1.04 & 122.0 & $2400(3 \times 800)$ \\
J 1044+0353a & 104457.8 & +035313 & 0.01274 & 51.2 & 1.19 & 101.5 & $3600(3 \times 1200)$ \\
J 1044+0353b & 104458.0 & +035313 & 0.01284 & 51.2 & 1.19 & 101.5 & $3600(3 \times 1200)$ \\
J 1201+0211 & 120122.3 & +021108 & 0.00327 & 14.0 & 1.21 & 119.0 & $2400(2 \times 1200)$ \\
J 1414-0208 & 141454.1 & -020823 & 0.00528 & 23.0 & 1.16 & 155.3 & $3600(3 \times 1200)$ \\
J 2230-0006 & 223036.8 & -000637 & 0.00559 & 24.9 & 1.33 & 156.0 & $3600(3 \times 1200)$ \\
J 2302+0049 & 230210.0 & +004939 & 0.03302 & 134.6 & 1.17 & 156.0 & $2400(2 \times 1200)$ \\
\hline
\end{tabular}

${ }^{a}$ Distance, derived after correction of the measured redshifts for the motion relative to the Local Group centroid and the Virgocentric flow, assuming a Hubble constant of $75 \mathrm{~km} \mathrm{~s}^{-1} \mathrm{Mpc}^{-1}$.

applicable to bright SDSS galaxies only and may be affected by large uncertainties compared with the standard method. In addition, some of the strongest emission lines in SDSS spectra are truncated or saturated. Moreover, the fibers used in the acquisition of SDSS spectra encompass a region of $3^{\prime \prime}$ in diameter and are typically positioned on the galaxy center. As a result, the SDSS data rarely enable spectroscopic studies of off-center SF regions. For this reason, follow-up spectroscopy of suspected XBCD candidates with off-center HII regions is much needed. This was demonstrated in e.g. Izotov et al. (2006b), who discovered the XBCD J2104-0035 by follow-up long-slit spectroscopy of selected SDSS galaxies. Star-forming activities in this system proceed mainly in a detached metal-poor $[12+\log (\mathrm{O} / \mathrm{H})=$ $7.26 \pm 0.03$ ] HII region $\sim 4^{\prime \prime}$ northeast of the geometrical center of the galaxy host, which was not included in the spectroscopic data set of SDSS.

The spectroscopic analysis presented here is supplemented by surface photometry. This is a step towards the systematization of the photometric and morphological properties of extremely metal-poor emission-line galaxies, which is one of the main goals of our studies.

\subsection{Data acquisition and reduction}

New spectroscopic observations of seven HII regions in six SDSS galaxies, and one 6dFGRS galaxy (g0405204-364859) were obtained on 10-13 April, and 6-8 October, 2005 with the EFOSC2 (ESO Faint Object Spectrograph and Camera) mounted at the $3.6 \mathrm{~m}$ ESO telescope at La Silla. The coordinates of the targets are listed in Table 1.

The observing conditions were photometric during all nights. For the spring observations, we used the grism \#14 and the grating $600 \mathrm{~g} / \mathrm{mm}$. The long-slit of dimensions $1^{\prime \prime} \times$ $300^{\prime \prime}$ was centered on the brightest part of each galaxy. The chosen instrumental setup allowed a wavelength coverage of $\lambda \lambda 3200-5083$, a spectral resolution of $\sim 6.2 \AA(F W H M)$, and a spatial scale of 0.314 pixel $^{-1}$ along the slit for the chosen $2 \times$ 2 pixel binning.

During the autumn observations, the grism \#07 and the grating $600 \mathrm{~g} / \mathrm{mm}$ were used, resulting in a wavelength coverage of $\lambda \lambda 3250-5200$. These observations were carried out with a 1 .' $2 \times 300^{\prime \prime}$ slit centered on the brightest part of each galaxy. The spectral resolution and spatial scale along this slit were $\sim 6.2 \AA$ $(F W H M)$ and $0 . ' 157$ pixel $^{-1}$, respectively.

All galaxies were observed at low airmass $(\$ 1.2)$ or along the parallactic angle, therefore no corrections for atmospheric refraction were applied. The total exposure per target ranged between 40 and 60 min and was split up in two to three subexposures to allow for a more efficient cosmic ray rejection. The seeing varied between $0 .{ }^{\prime} 8$ and $11^{\prime \prime} 5$. Three spectrophotometric standard stars were observed during each night for flux calibration. The journal of the observations is given in Table 1 .

In Fig. 1, we reproduce the slit position on optical CCD images. The morphology of the galaxies is illustrated using stacked acquisition exposures (panel b) or SDSS gri images (panels a and $\mathrm{c}-\mathrm{g}$ ). In Table 1 , we list the adopted distance to each target. Distances were derived after correction of the measured redshifts for the motion relative to the Local Group centroid and the Virgocentric flow (Kraan-Korteweg 1986) and assuming a Hubble constant of $75 \mathrm{~km} \mathrm{~s}^{-1} \mathrm{Mpc}^{-1}$.

We note that the slit used is in all cases narrower than the isophotal size of the star-forming component $R_{\mathrm{SF}}$ (cf. Col. 4 in Table 4). Given that the intensity profile of the SF component (Fig. 3) is strongly centrally peaked with an effective radius $r_{\text {eff,SF }} \lesssim 1^{\prime \prime}$ (Table 4, Col. 12), our long-slit spectra include in all but one case (J1414-0208) most of its emission. Therefore, our results are not affected significantly by aperture effects, which, for more distant targets, can dilute the equivalent widths $(E W s)$ of emission lines due to inclusion of the adjacent stellar background of the LSB host galaxy. This is probably also the case for g0405204-364859, the $r_{\text {eff,SF }}$ of which was estimated from stacked acquisition exposures to be $<2$ ". However, given that in several BCDs nebular emission is more extended than $R_{\mathrm{SF}}$ (P02), one cannot exclude that emission line fluxes and $E W \mathrm{~s}$ are slightly underestimated.

The $3.6 \mathrm{~m}$ spectra were reduced with the IRAF ${ }^{1}$ software package. This includes bias-subtraction, flat-field correction, cosmic-ray removal, wavelength calibration, night sky background subtraction, correction for atmospheric extinction, and absolute flux calibration of each two-dimensional spectrum. We extracted one-dimensional spectra from the brightest part of each galaxy studied. The spectra were corrected for interstellar extinction using the reddening curve by Whitford (1958) and transformed into the rest-frame based on the redshift $z$, derived from several bright emission lines. The redshift-corrected onedimensional spectra of the 8 selected HII regions are shown in Fig. 2.

\footnotetext{
${ }^{1}$ IRAF is the Image Reduction and Analysis Facility distributed by the National Optical Astronomy Observatory, which is operated by the Association of Universities for Research in Astronomy (AURA) under cooperative agreement with the National Science Foundation (NSF).
} 

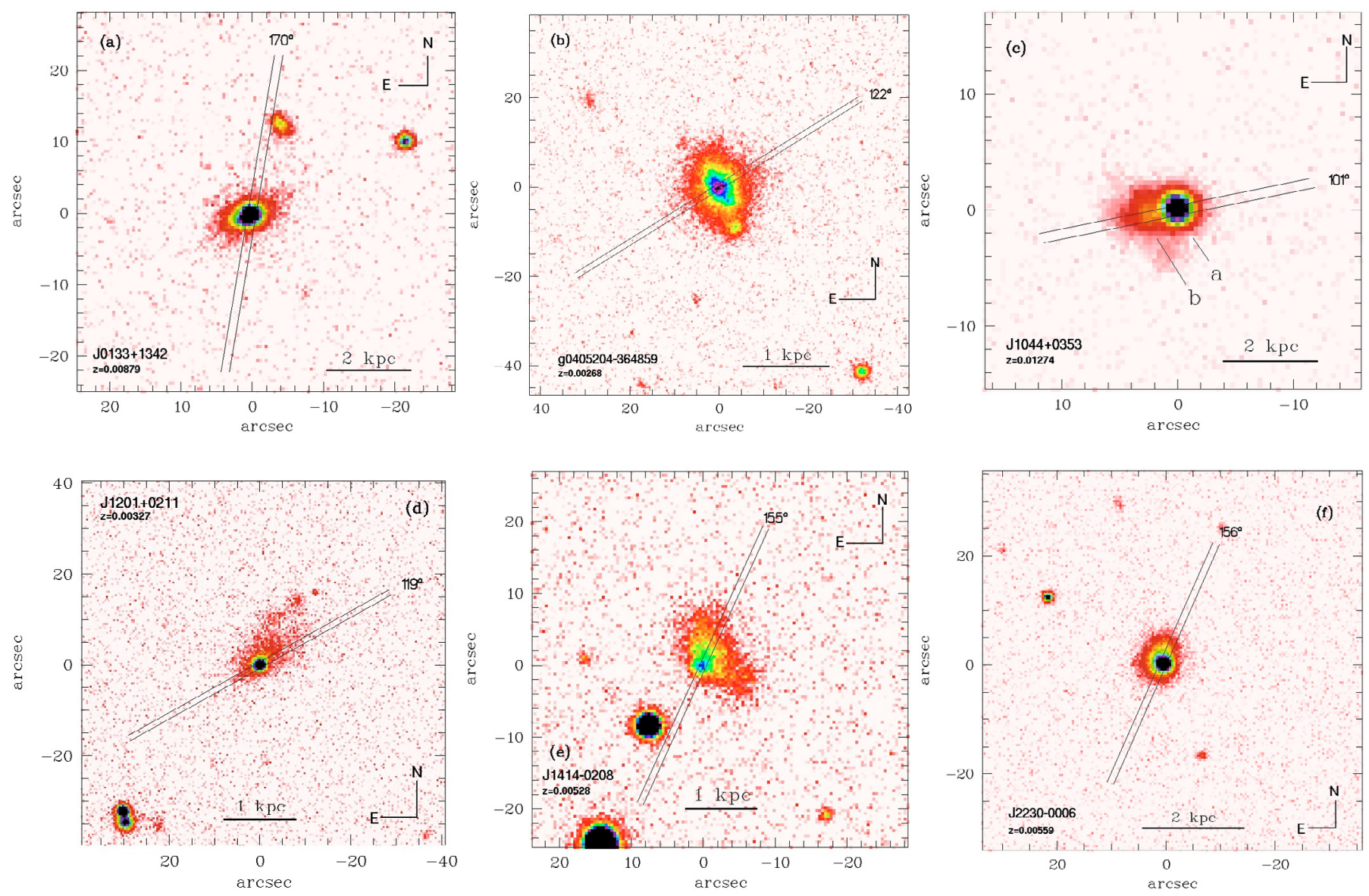

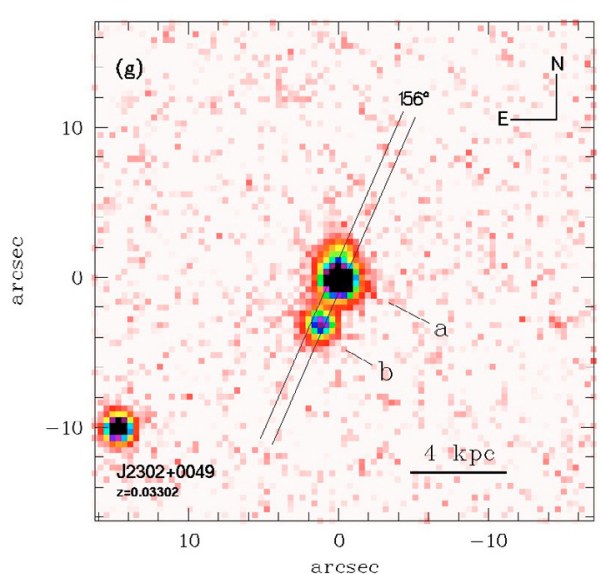

Emission line fluxes were measured using Gaussian profile fitting. The errors in the line fluxes were calculated from the photon statistics of the non-flux-calibrated spectra. These were then propagated in the calculations of the elemental abundance errors.

The observed fluxes of all hydrogen Balmer lines except for the $\mathrm{H} 7$ and $\mathrm{H} 8$ lines were used to determine the interstellar extinction and the underlying stellar absorption. The two excluded lines were blended with other strong emission lines and could not therefore be used for the determination of interstellar reddening.

The extinction coefficient $C(\mathrm{H} \beta)$ and the equivalent width of absorption hydrogen lines $E W_{\text {abs }}$ were derived by minimising the deviations in the corrected hydrogen emission line fluxes from the theoretical recombination values. In this procedure, we assumed that $E W_{\text {abs }}$ is the same for all hydrogen lines. The observed emission line fluxes $F(\lambda)$ relative to the $\mathrm{H} \beta$ fluxes and those corrected for interstellar extinction and underlying stellar absorption $I(\lambda)$ relative to the $\mathrm{H} \beta$, equivalent widths $E W$ of emission lines, extinction coefficients $C(\mathrm{H} \beta)$, observed $\mathrm{H} \beta$ fluxes $F(\mathrm{H} \beta)$, and equivalent widths of the hydrogen absorption lines are listed in Table 2.

\section{Results}

\subsection{Chemical abundances}

The electron temperature $T_{\mathrm{e}}$ and the ionic and total heavy element abundances were derived following Izotov et al. (2006a). In particular, for the $\mathrm{O}^{2+}$ and $\mathrm{Ne}^{2+}$ ions, we adopted the temperature $T_{\mathrm{e}}(\mathrm{O}$ III $)$ derived from the $[\mathrm{O}$ III] $\lambda 4363 /(\lambda 4959+\lambda 5007)$ emission line ratio. The $\mathrm{O}^{+}$and $\mathrm{Fe}^{++}$abundances were derived with the temperature $T_{\mathrm{e}}(\mathrm{O}$ II), which was obtained from the relation between $T_{\mathrm{e}}(\mathrm{O}$ III $)$ and $T_{\mathrm{e}}(\mathrm{O}$ II) of Izotov et al. (2006a). 


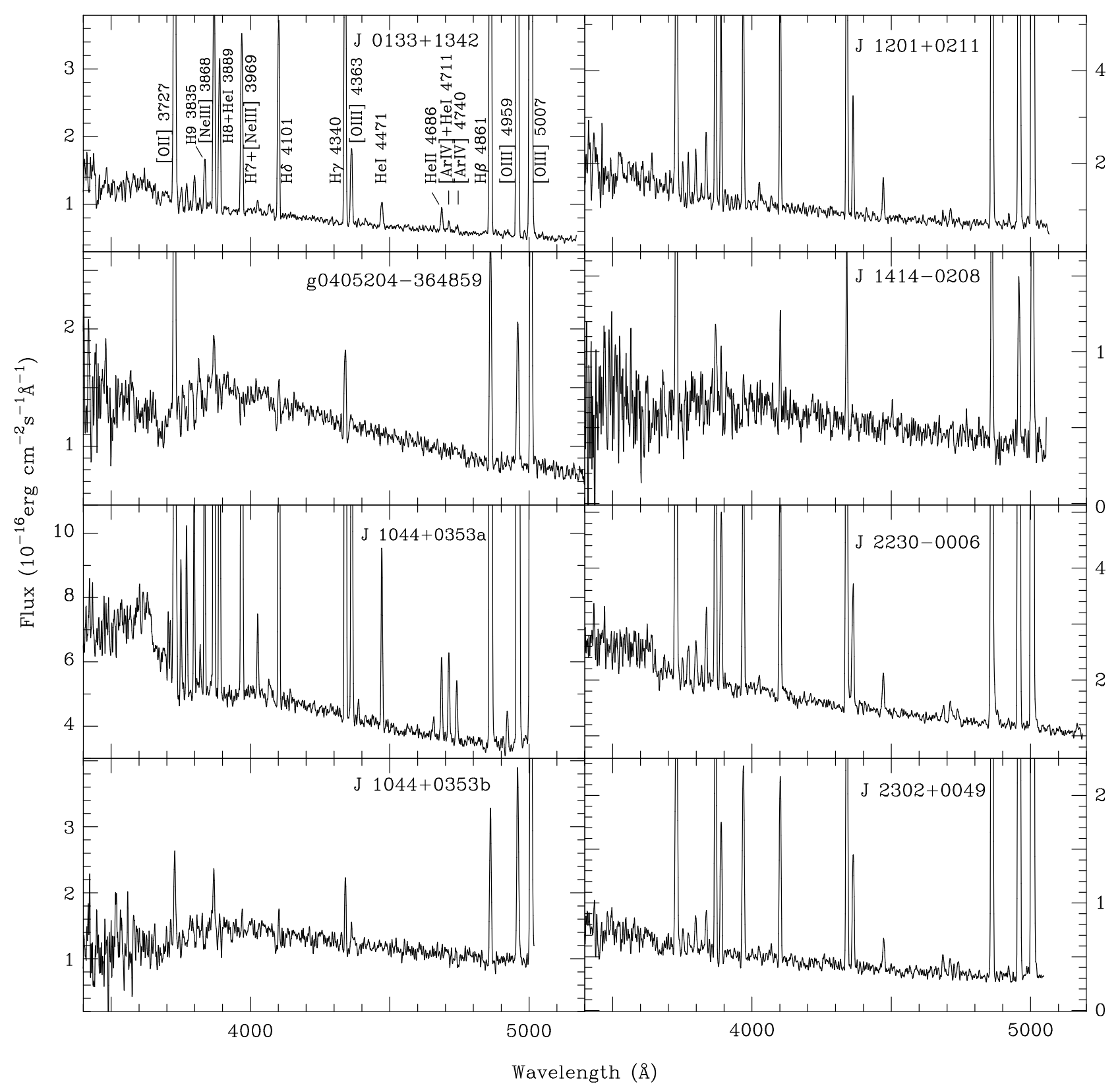

Fig. 2. Redshift- and extinction-corrected spectra of 8 HII regions in the metal-poor galaxies studied with the ESO $3.6 \mathrm{~m}$ telescope. Line identifications are shown for the first galaxy in our list.

The $3.6 \mathrm{~m}$ telescope spectra covered the blue wavelength region, thus the [S II] $\lambda 6717,6731$ emission lines, usually used for the determination of the electron number density, were not observed. Therefore for abundance determinations, we adopted $N_{\mathrm{e}}=100 \mathrm{~cm}^{-3}$. The derived abundances are insensitive to the precise value of the electron number density in the low-density limit $\left(N_{\mathrm{e}} \lesssim 10^{3} \mathrm{~cm}^{-3}\right)$, which is applicable for the $\mathrm{H}$ II regions considered here.

The electron temperatures $T_{\mathrm{e}}(\mathrm{O}$ III $)$ and $T_{\mathrm{e}}(\mathrm{O}$ II $)$ for the high and low-ionization zones in $\mathrm{H}$ II regions, respectively, the ionization correction factors $(I C F \mathrm{~s})$, and the ionic and total heavy element abundances for oxygen, and, where possible, neon and iron are listed in Table 3.

The derived oxygen abundance $12+\log (\mathrm{O} / \mathrm{H})$ of the sample galaxies is in the range between 7.3 and $~ 7.6$, the objects investigated here therefore belong to the rare class of extremely metaldeficient emission-line galaxies with an oxygen abundance $\$ 7.6$
(Thuan \& Izotov 2005). The $\mathrm{N} / \mathrm{O}$ and $\mathrm{Fe} / \mathrm{O}$ abundance ratios are consistent within the errors with the ratios obtained for other metal-deficient galaxies (Izotov \& Thuan 1999).

Comparison of $3.6 \mathrm{~m}$ and SDSS data for the common three HII regions shows that the $3.6 \mathrm{~m}$ oxygen abundances are lower by $\sim 0.04$ dex than those in the papers of Kniazev et al. (2003, 2004). These relatively small discrepancies can be explained by differences in the data acquisition (e.g. telescope, sky transparency) and methods employed in the chemical abundance determination.

It is worth pointing out that all known XBCDs with oxygen abundance determinations in multiple positions over their optical extent show little, if any, abundance variations. Therefore, the oxygen abundance derived in one HII region of these systems may be considered to within $\sim 0.1$ dex as representative of the ionized interstellar medium (ISM) over the entire galaxy. Examples are SBS 0335-052E, which differs by less than 0.1 dex 


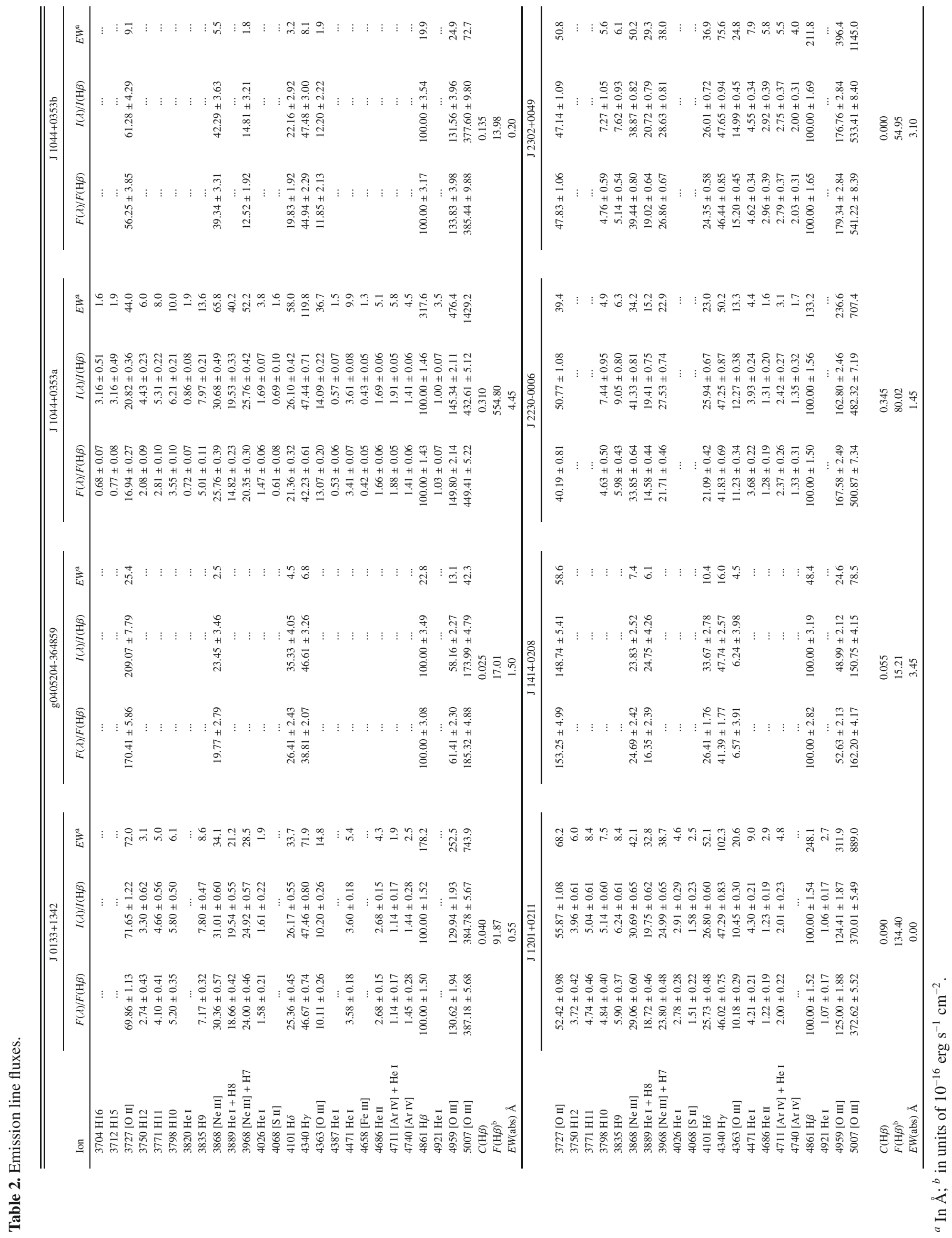


in the oxygen abundance of its central SF clusters \#1, 2 and its peripheral SF region \#7 (cf. Izotov et al. 1997b; Papaderos et al. 2006a), and I Zw 18 of essentially equal oxygen abundance in both its northwestern and southeastern SF region (Izotov et al. 1999). Several other examples may be found in e.g. Izotov \& Thuan (2007).

\subsection{Photometric analysis}

\subsubsection{Derivation and general properties of surface brightness profiles}

Surface brightness profiles (SBPs) for the SDSS galaxies were derived using method iv introduced in P02. This SBP determination algorithm was developed especially for the study of irregular SF galaxies, presenting strong intensity and colour gradients, and has been successfully applied both to nearby BCDs (P02; Noeske et al. 2003) and compact SF galaxies at intermediate redshift (Noeske et al. 2006).

Surface photometry was carried out in the $g$ and $i$ bands. As is evident from Fig. 3, SBPs reach in all cases a surface brightness of $\sim 26 \mathrm{gmag} / \square^{\prime \prime}$, i.e. they allow the detection and quantitative study of the faint and more extended LSB host galaxy underlying the SF component. It is well established from earlier work (P96b) that the host dominates the line-of-sight intensity for $\mu \gtrsim 24.5 B \mathrm{mag} / \square^{\prime \prime}$. This is also true for dwarf irregulars (dIs), which, typically, do not show an appreciable SF activity in their LSB periphery (Patterson \& Thuan 1996; Makarova \& Karachentsev 1998; Youngblood \& Hunter 1999; van Zee 2000).

The SBPs of most sample galaxies reveal a two-component structure that is typical of BCDs i.e. an exponential slope at large radii which characterises the LSB emission, and a central luminosity excess, which can be attributed to the SF component. These two distinct stellar populations are also reflected in colour maps (Fig. 4) and colour profiles (Fig. 3), which display a considerable colour contrast between the SF center and the LSB periphery (cf. J0133+1342, J1201+0211, J2230-0006), as typically found for BCDs (P96b; P02; Cairós et al. 2001b; Gil de Paz \& Madore 2005). The only galaxy in our sample undergoing weak SF activity with a comparatively low $E W(\mathrm{H} \beta)$ $(\sim 50 \AA)$ and an almost exponential SBP all the way to its center is J1414-0208. Not surprisingly, this system also exhibits negligible colour gradients and subtle colour variations of an amplitude less than 0.4 mag.

SBPs were decomposed into separate profiles representing the luminosity of the SF and the LSB components following the procedure described in $\mathrm{P} 02$. These two components are shown in each SBP (Fig. 3) as solid and dashed lines, respectively. We chose not to apply the 3-component scheme used in P96a, which consists of an exponential, Sérsic, and Gaussian component, which represent the host galaxy and the SF component at both intermediate and small radii, respectively.

This was mainly because the central Gaussian component does not correlate with other galactic structural properties and is always far narrower than the isophotal radius of the SF component, termed by $\mathrm{P} 96 \mathrm{a}$ as plateau radius $R_{\mathrm{SF}}$. Instead, we consider only the total emission from the SF component, i.e. the emission in excess of the exponential fit to the LSB host.

In all but one of the SDSS galaxies studied, the LSB host was approximated by an exponential fitting function of the form:

$I\left(R^{*}\right) \equiv I_{\exp }=I_{0} \exp \left(-\frac{R^{*}}{\alpha}\right)$ 

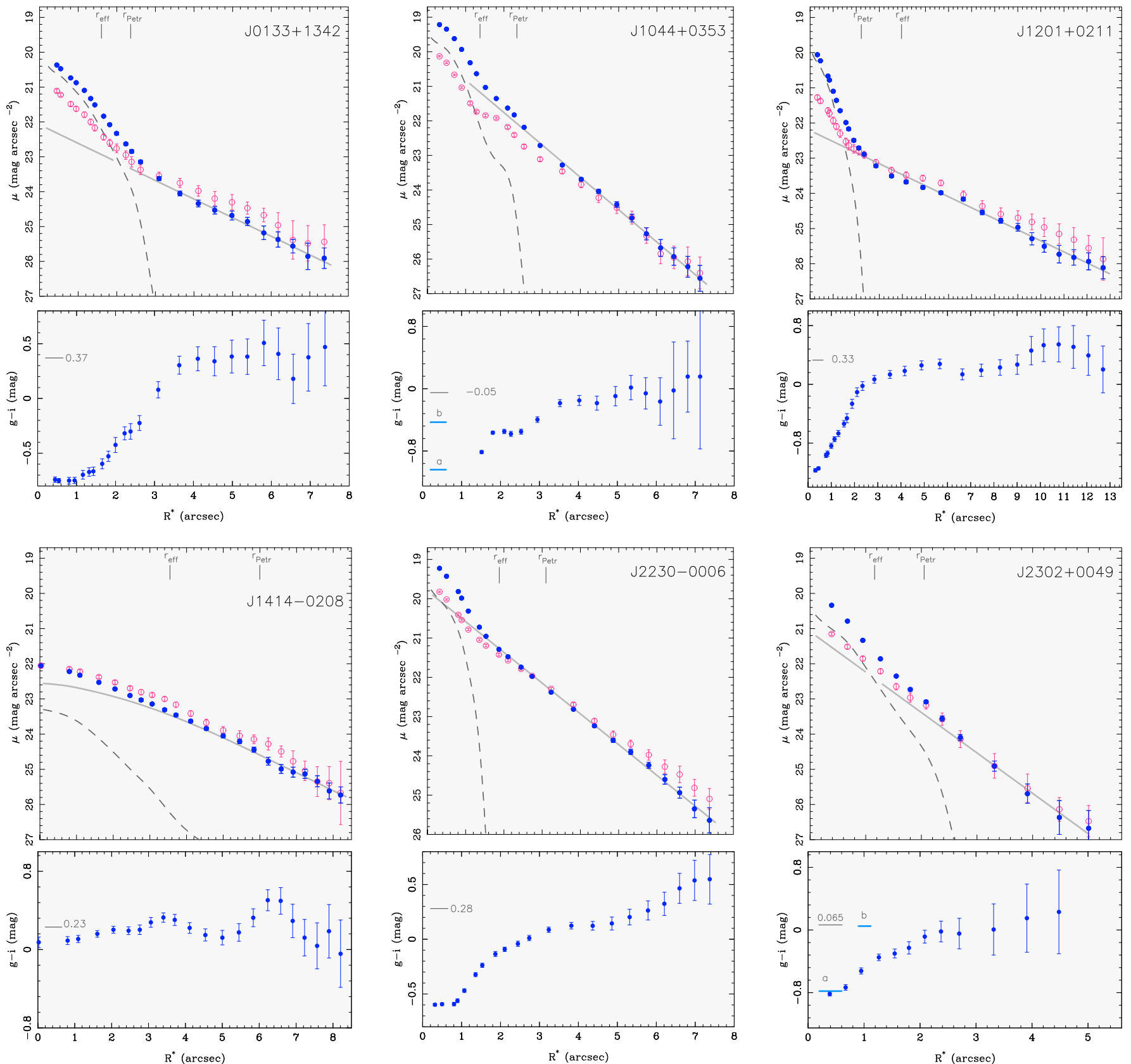

Fig. 3. Surface brightness profiles (SBPs) of the SDSS sample galaxies (upper panels) in $g$ (full circles) and $i$ (open circles). Solid lines show fits to the LSB host galaxy (Table 4) according to Eqs. (2) and (1) for J1414-0208 and all other objects, respectively. The surface brightness distribution of the SF component in the $g$ band is shown by dashed curves. The effective $r_{\text {eff }}$ and Petrosian $r_{\text {Petr }}$ radii of each galaxy are indicated by vertical lines. $g-i$ colour profiles (lower panels) have been computed by subtraction of the $i$ SBPs from the $g$ SBPs. The mean $g-i$ colour of the LSB host galaxy within the fitting range (Col. 10 in Table 4) is indicated by the horizontal lines at the left part of each diagram. The mean colours of the regions $\mathrm{a}$ and $\mathrm{b}$ in $\mathrm{J} 1044+0353$ and $\mathrm{J} 2302+0049$ are depicted by horizontal lines on the left-hand side of the corresponding diagrams.

where $I_{0}$ and $\alpha$ denote the central intensity and the exponential scale length of the host, and $R^{\star}$ is the photometric radius.

In the case of J1414-0208, Eq. (1) is unsuitable because at intermediate radii it predicts a higher intensity than observed. Evidently, the outer exponential profile of the LSB component cannot continue all the way to $R^{\star}=0^{\prime \prime}$ but probably flattens at small radii. Therefore, following P96a, we fitted the LSB host of J1414-0208 with a modified exponential model involving a mild central flattening of the form:

$I\left(R^{*}\right)=I_{\exp } \cdot\left[1-\epsilon_{1} \exp \left(-P_{3}\left(R^{*}\right)\right)\right]$, where $P_{3}\left(R^{*}\right)$ is defined as

$P_{3}\left(R^{*}\right)=\left(\frac{R^{*}}{\epsilon_{2} \alpha}\right)^{3}+\left(\frac{R^{*}}{\alpha} \frac{1-\epsilon_{1}}{\epsilon_{1}}\right)$.

For radii $R^{\star} \lesssim \epsilon_{2} \cdot \alpha$, the distribution given by Eq. (2) depends on the central depression $\epsilon_{1}=\Delta I / I_{0}$ relative to the pure exponential profile $I_{\text {exp }}$. Given that $\epsilon_{2}$ is typically $\approx 3 \cdot \epsilon_{1}$ (cf., Fricke et al. 2001; Noeske et al. 2003, 2005), Eq. (2) can be simplified to a 3-parameter formula. In the case of J1414-0208, we adopt a moderate central flattening $\epsilon_{1}=0.6$, which corresponds to a 

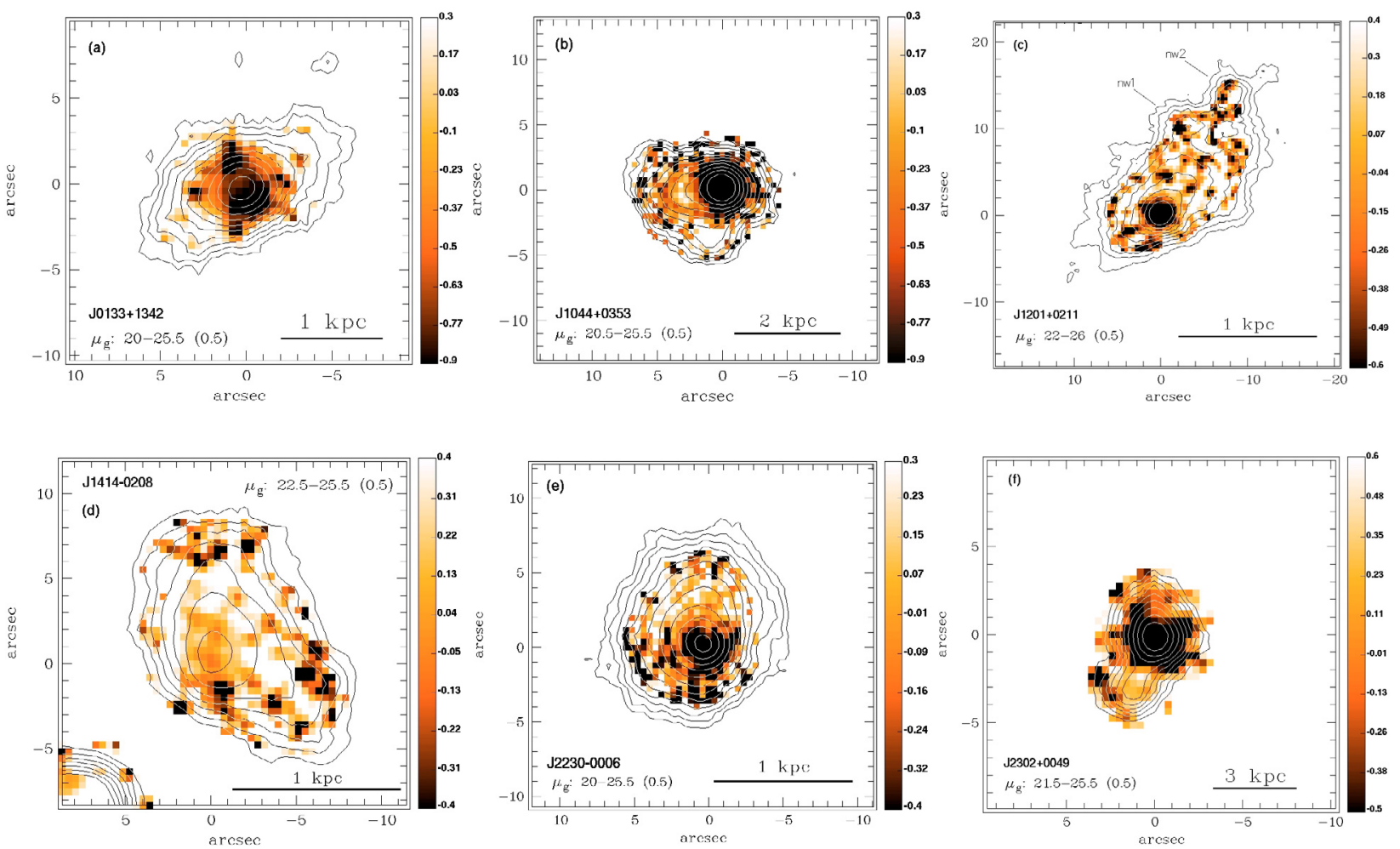

Fig. 4. $g-i$ colour maps of the SDSS sample galaxies displayed in the colour interval indicated by the vertical bars to the right of each panel. The surface brightness range and the spacing of the superposed $g$ band contours are indicated. North is to the top and east to the left.

Table 4. Structural properties of the SDSS sample galaxies in the $g$ band.

\begin{tabular}{|c|c|c|c|c|c|c|c|c|c|c|c|}
\hline Name & $\begin{array}{l}\mu_{\mathrm{E}, 0} \\
\alpha \\
(2) \\
\end{array}$ & $\begin{array}{c}M_{\mathrm{SF}} \\
M_{\text {host }} \\
\text { (3) }\end{array}$ & $\begin{array}{c}R_{\mathrm{SF}} \\
R_{\text {host }} \\
(4) \\
\end{array}$ & $\begin{array}{l}m \\
M \\
(5) \\
\end{array}$ & $\begin{array}{c}r_{\text {eff }} \\
\mu_{\text {eff }} \\
(6)\end{array}$ & $\begin{array}{c}r_{\text {Petr }} \\
m_{\text {Petr }} \\
(7) \\
\end{array}$ & $\begin{array}{c}C I \\
\eta \\
(8) \\
\end{array}$ & $\begin{array}{c}R_{\text {host }} / r_{\text {eff }} \\
l_{\mathrm{SF}}\left(R_{\text {host }}, \text { total }\right) \\
(9) \\
\end{array}$ & $\begin{array}{c}\text { fit range } \\
(g-i)_{\text {host }} \\
(10) \\
\end{array}$ & $\begin{array}{c}t \text { (SFH1, 2) } \\
M_{\star}(\text { SFH } 1,2) \\
(11) \\
\end{array}$ & $\begin{array}{c}r_{\mathrm{eff}, \mathrm{SF}} \\
m-M \\
(12) \\
\end{array}$ \\
\hline \multirow{2}{*}{ J $0133+1342$} & $22.1 \pm 0.1$ & -14.2 & 0.47 & 17.9 & 0.30 & 0.43 & 0.74 & 3.13 & $33^{\prime \prime} 9-7^{\prime \prime} .2$ & $1.0,2.7$ & 1.1 \\
\hline & $0.37 \pm 0.02$ & -14.3 & 0.93 & -15.0 & 20.98 & 18.4 & 2.25 & $0.47,0.53$ & $0.37 \pm 0.09$ & $1.9,3.0$ & 32.887 \\
\hline \multirow[t]{2}{*}{ J 1044+0353 } & $19.9 \pm 0.2$ & -15.0 & 0.60 & 17.2 & 0.35 & 0.57 & 0.78 & 3.63 & $3 . .2-7{ }^{\prime \prime} .5$ & $0.1,1.0$ & 0.9 \\
\hline & $0.29 \pm 0.01$ & -15.9 & 1.27 & -16.3 & 19.9 & 17.5 & 1.4 & $0.69,0.70$ & $-0.05 \pm 0.13$ & $0.4,2.2$ & 33.538 \\
\hline \multirow[t]{2}{*}{ J 1201+0211 } & $22.2 \pm 0.1$ & -11.7 & 0.14 & 17.4 & 0.27 & 0.15 & 0.92 & 2.00 & 4 4.' $6-13 " .^{\prime \prime}$ & $1.0,2.5$ & 0.9 \\
\hline & $0.23 \pm 0.01$ & -12.7 & 0.54 & -13.3 & 22.4 & 18.5 & 5.2 & $0.72,0.79$ & $0.33 \pm 0.14$ & $1.9,2.5$ & 30.731 \\
\hline \multirow[t]{2}{*}{ J 1414-0208 } & $21.6 \pm 0.1$ & -10.8 & 0.24 & 18.1 & 0.38 & 0.67 & 0.87 & 1.80 & $3^{\prime \prime}-8 .{ }^{\prime \prime} 4$ & $0.6,2.0$ & $1 . \prime 8$ \\
\hline & $0.24 \pm 0.01$ & -13.4 & 0.69 & -13.7 & 22.8 & 18.3 & 0.89 & $0.92,0.94$ & $0.23 \pm 0.1$ & $0.4,0.7$ & 31.809 \\
\hline \multirow[t]{2}{*}{ J 2230-0006 } & $19.8 \pm 0.1$ & -13.0 & 0.19 & 17.0 & 0.23 & 0.37 & 0.94 & 3.36 & $3^{\prime \prime}-7{ }^{\prime \prime} 7$ & $0.8,2.2$ & 0.7 \\
\hline & $0.17 \pm 0.01$ & -14.8 & 0.75 & -15.0 & 20.33 & 17.31 & 1.45 & $0.84,0.85$ & $0.28 \pm 0.15$ & $2.3,2.9$ & 31.981 \\
\hline \multirow[t]{2}{*}{ J $2302+0049$} & $20.8 \pm 0.3$ & -15.9 & 1.33 & 18.6 & 0.73 & 1.33 & 0.58 & 2.85 & $2 . .4-5 . .2$ & $0.3,1.4$ & 0.8 \\
\hline & $0.58 \pm 0.03$ & -16.4 & 2.09 & -17.0 & 20.86 & 18.87 & 1.37 & $0.63,0.65$ & $0.07 \pm 0.2$ & $1.8,4.8$ & 35.645 \\
\hline
\end{tabular}

central surface brightness and total magnitude that are 0.55 mag and 0.2 mag fainter, respectively, than those predicted by fitting Eq. (1).

\subsubsection{Spatial extent of the star-forming component and the LSB host}

One of our principle aims is to use surface photometry to determine the structural properties of BCDs/XBCDs and study the influence of the gravitational potential of their LSB host on their star formation process (see also P96b). For this purpose, it is important to first disentangle the LSB and the SF component and determine their respective mass fractions and spatial extents. To this end, P96a introduced the isophotal radii $R_{\text {host }}$ and $R_{\mathrm{SF}}$ of these two components at an extinction-corrected surface brightness level of $25 \mathrm{Bmag} / \square^{\prime \prime}$.

A practical advantage of $R_{\mathrm{SF}}$ is that it can be easily derived from a 2-component SBP decomposition and shows little dependence on the luminosity of the SF component, which in BCDs may strongly vary on a timescale of a few $10^{7} \mathrm{yr}$.

This is not the case for either the effective radius or concentration indices evaluated from the radii enclosing different fractions of the total flux (for example, the ratio $\log \left(R_{80} / R_{20}\right)$ of the radii containing $80 \%$ and $20 \%$ of the total light). These quantities depend primarily on the luminosity rather than on the spatial extent of the SF component (see e.g. P96a; and Barton \& van Zee 2001; P06). 


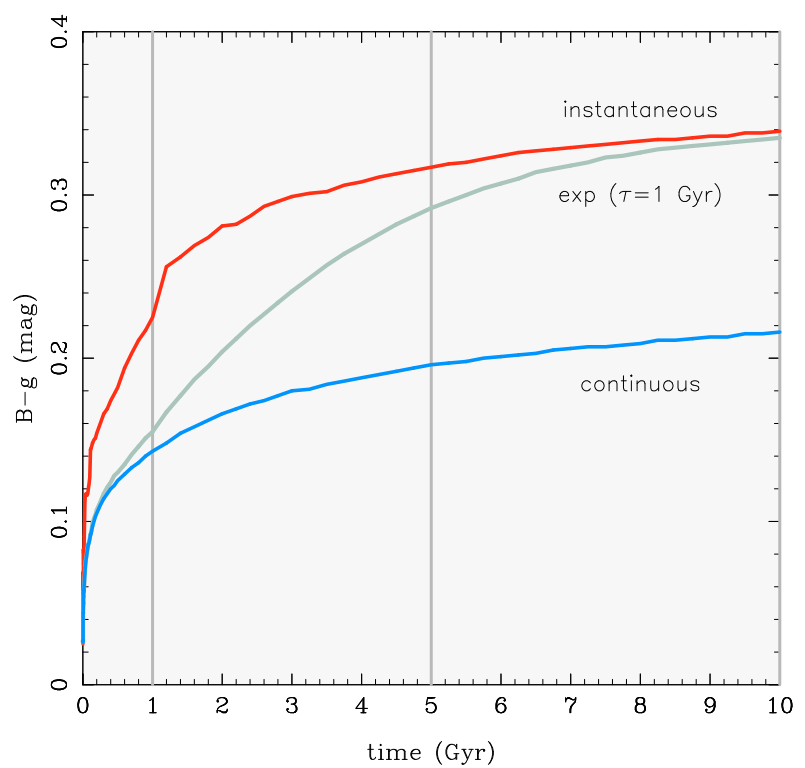

Fig. 5. Evolution of the $B-g$ colour index as a function of time for three star formation histories: SFH1 (instantaneous), SFH2 (exponentially decreasing star formation rate with an $e$-folding time of $1 \mathrm{Gyr}$ ) and SFH3 (continuous, constant star formation rate).

To be able to compare directly the photometric properties, e.g., $R_{\mathrm{SF}}, R_{\text {host }}$ and magnitudes, of the present galaxy sample in the SDSS $g$ with the available literature data in $B$ it is necessary to first infer the $g$ surface brightness that corresponds to a $B$ surface brightness of $25 \mathrm{mag} / \square^{\prime \prime}$. For this, we study the time evolution of the $B-g$ index for three different star formation histories (SFHs): an instantaneous SF episode (SFH1), an exponentially decreasing star formation rate with an $e$-folding timescale of $1 \mathrm{Gyr}$ (SFH2), and a constant, continuous SF (SFH3). Calculations were carried out with the PEGASE 2.0 code (Fioc \& Rocca-Volmerange 1997) and refer to a stellar population with a fixed stellar metallicity of $Z=0.001$ and a Salpeter initial mass function with lower and upper mass limits of 0.1 and $100 M_{\odot}$, respectively.

Figure 5 shows that the $B-g$ index for SFH1 and SFH2 differs by less than 0.1 mag after $t \sim 1 \mathrm{Gyr}$, and that both SFHs imply a $B-g \approx 0.3 \mathrm{mag}$ for $t \geq 5$ Gyr. Since these two models are probably the most appropriate approximations of the SFH of the SF component and that of the LSB host, we assume in the following the relation $m_{B} \approx m_{g}+0.3$.

\subsubsection{Photometric properties of the sample galaxies}

Table 4 lists photometric quantities for the six SDSS galaxies in our sample. All tabulated values are corrected for Galactic absorption based on the extinction maps by Schlegel et al. (1998) available in the $\mathrm{NED}^{1}$. Column 2 of Table 4 gives the extrapolated central surface brightness $\mu_{\mathrm{E}, 0}\left(\mathrm{mag} / \square^{\prime \prime}\right)$ and the exponential scale length $\alpha$ (kpc) of the LSB host galaxy, obtained by fitting Eq. (1) to the outer part of each SBP. In Col. 3, we tabulate the absolute $g$ band magnitude of the SF component $M_{\mathrm{SF}}$ and that of the LSB host $M_{\text {host }}$ within their respective isophotal radii $R_{\mathrm{SF}}$ and $R_{\text {host }}$ (Col. 4) at a surface brightness level of $24.7 \mathrm{~g} \mathrm{mag} / \square^{\prime \prime}$ (see discussion in Sect. 3.2.2). In Col. 5, we

\footnotetext{
1 NASA/IPAC Extragalactic Database;

http://nedwww . ipac . caltech. edu
}

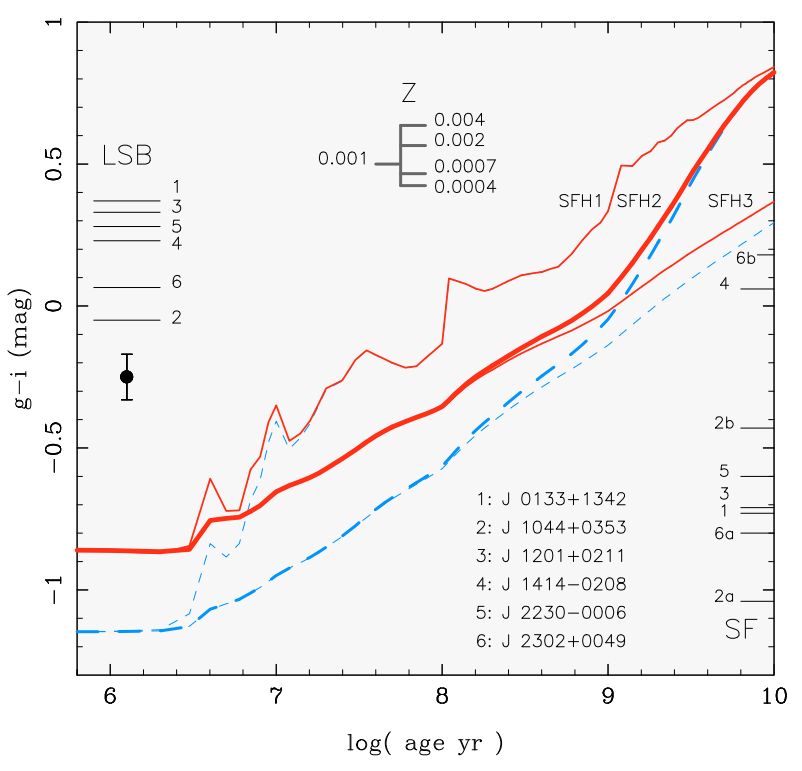

Fig. 6. Evolution of the $g-i$ colour index as a function of time for the star formation scenarios SFH1 through SFH3 (Sect. 3.2.2) based on Pegase 2.0 models involving purely stellar emission (solid lines) as well as stellar and ionized gas emission (dashed lines). The average colour shift within $1 \leq t(\mathrm{Gyr}) \leq 3$ that a variation in the stellar metallicity between 0.0004 and 0.004 would produce is indicated. Horizontal lines on the left-hand side of the diagram indicate the mean $g-i$ colour of the LSB host within the fitted radius range (Table 4, Col. 10) along with a typical $1 \sigma$ uncertainty. Likewise, the measured $g-i$ colour in the SF component is depicted by horizontal lines at the r.h.s. part of the diagram.

tabulate the apparent and absolute magnitude of each galaxy as derived from SBP integration. Column 6 lists the effective radius $r_{\mathrm{eff}}(\mathrm{kpc})$ and the mean surface brightness $\mu_{\mathrm{eff}}$ inside $r_{\mathrm{eff}}$, and in Col. 7 we list the Petrosian radius $r_{\text {Petr }}(\mathrm{kpc})$, which is defined to be the radius at which the Petrosian $\eta$ function (Petrosian 1976) decreases to a value of $1 / 3$ (see e.g. Takamiya 1999), and the apparent magnitude $m_{\text {Petr }}$ within $r_{\text {Petr }}$.

The concentration index $C I$, defined by P96a to be $1-\left(R_{\mathrm{SF}} / R_{\text {host }}\right)^{2}$ is tabulated in Col. 8. This quantity measures the fractional area of the SF component and attains a maximal value for systems containing a single, compact SF region. In the same column, we list the Sérsic exponent $\eta$ obtained from fitting a Sérsic model of the form $I_{0} \exp \left(-R^{\star} / \alpha\right)^{1 / \eta}$ to each $g$ SBP. Column 9 lists the ratio $R_{\text {host }} / r_{\text {eff }}$ and the luminosity contribution $l_{\mathrm{SF}}$ of the host galaxy both within $R_{\text {host }}$ and with regard to the total galaxy emission. In Col. 10, we tabulate the fitted radius range of the LSB host and the mean $g-i$ colour within that radius interval. The age of the host galaxy in Gyr, estimated from its colour using PEGASE 2.0 models for SFH1 and SFH2, and its corresponding present stellar mass in $10^{7} M_{\odot}$ are given in Col. 11. Column 12 lists the effective radius $r_{\mathrm{eff}, \mathrm{SF}}$ of the SF component in arcsec and the distance modulus $m-M$ of each target.

In Fig. 7, we plot the stellar mass $M_{\star}$ of the LSB host of our sample galaxies, derived on the basis of their star formation histories SFH1 and SFH2 and oxygen abundance. A clear trend (Spearman's rank correlation coefficient: 0.83 ) of the form

$12+\log (\mathrm{O} / \mathrm{H})=(4.2 \pm 0.6)+(0.45 \pm 0.09) \log _{10} M_{\star}$

between these two quantities is evident for masses derived adopting SFH2. The derived slope between gas-phase metallicity and stellar mass, $Z \propto M_{\star}^{0.45 \pm 0.09}$, is in good agreement with the relation $Z \propto M_{\star}^{0.4}$ between stellar metallicity and mass predicted 


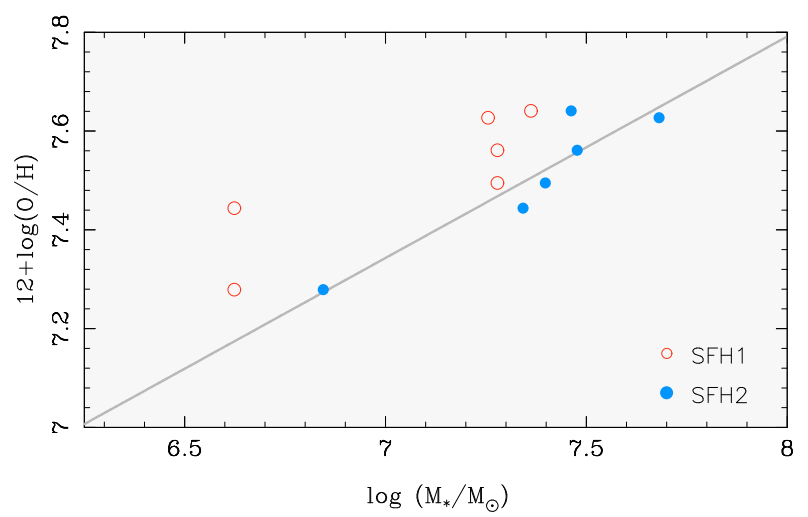

Fig. 7. Relation between the oxygen abundance $12+\log (\mathrm{O} / \mathrm{H})$ and the stellar mass $M_{\star}$ of the LSB host of our SDSS sample galaxies. Stellar masses were derived using Pegase 2.0 models on the base of the star formation histories SFH1 (open symbols) and SFH2 (filled symbols). The diagonal line shows a linear fit to the oxygen abundance for stellar masses estimated assuming SFH2.

by Dekel \& Woo (2003) and slightly steeper than the empirical relation $Z \propto M_{\star}^{0.3}$ inferred by Lee et al. (2006) from the integrated $4.5 \mu$ m luminosity of nearby dwarf irregular galaxies.

\section{Remarks on individual galaxies}

J0133+1342: this system contains an unresolved high-surface brightness SF region in the central part of a moderately irregular LSB host. The absolute $g$ magnitude of the host, $M_{g} \approx$ $-14.3 \mathrm{mag}$, translating to a $B$ band magnitude $M_{B} \approx-14 \mathrm{mag}$, places $\mathrm{J} 0133+1342$ well within the range of dwarf galaxies. The central surface brightness $\left(\mu_{0} \approx 22 \mathrm{mag} / \square^{\prime \prime}\right)$ and exponential scale length $(\alpha=0.37 \mathrm{kpc})$ of the host are typical of BCDs. This is also the case for the isophotal radius $\left(R_{\mathrm{SF}} \approx 0.5 \mathrm{kpc}\right)$ and the fractional luminosity of the SF component $(\sim 0.5)$. The blue $g-i$ colour $(-0.73 \mathrm{mag})$ within the central $3^{\prime \prime} \times 3^{\prime \prime}$ of the SF component indicates a substantial young stellar population. This is also suggested by the strong nebular emission of J0133+1342 with an $E W$ of $178 \AA$ and $743 \AA$ for the $\mathrm{H} \beta$ and [O III] $\lambda 5007$ lines, respectively. In agreement with the general trend observed for BCDs, this galaxy shows a gradual colour increase with increasing galactocentric radius and an almost constant, reddish colour in its LSB host.

In Fig. 6, we compare the colours of J0133+1342 with the expected $g-i$ colour evolution for the star formation scenarios SFH1 through to SFH3. In addition to models involving purely stellar emission (solid curves), we show models including ionized gas emission (dashed curves). The observed colours in the LSB host and SF component of the SDSS galaxies studied are depicted by horizontal lines in the left-hand side and right-hand side of the figure, respectively. From Fig. 6, it can be seen that the mean LSB colour of $0.37 \pm 0.08$ mag for $R^{\star} \geq 0.7 \mathrm{kpc}$ translates into an age of between 1 and $2.5 \mathrm{Gyr}$ for SFH1 and SFH2, respectively, suggesting a moderately evolved stellar population. The blue colours of the SF component correspond to a burst age of between 3 and 7 Myr (SFH1 assumed) with values in the lower range preferable because of the non-detection of Wolf-Rayet spectral features for this system.

The oxygen abundance that we derive from the $3.6 \mathrm{~m} \mathrm{ESO}$ telescope spectra, $12+\log (\mathrm{O} / \mathrm{H})=7.56 \pm 0.01$, compares well with the value of $7.60 \pm 0.03$ inferred by Kniazev et al. (2003) from SDSS spectra, and with the value of $12+\log (\mathrm{O} / \mathrm{H})=7.55 \pm$ 0.04 that we derive from the same data following the prescriptions of Izotov et al. (2006a).

g0405204-364859: this nearby system ( $D \approx 11 \mathrm{Mpc})$, identified in the 6dFGRS survey, has never been studied previously. Because of its southern declination, it was not included in the SDSS survey, and archival data also do not exist; no quantitative statements can therefore be made about its photometric structure. On the coadded acquisition exposure (90 s in $V$; Fig. 1), it appears to be a compact source with an overall regular morphology and elliptical LSB host with a possible faint extention in its southwestern direction.

The spectrum of this system is of low signal-to-noise ratio, yet clearly reveals weak emission lines superimposed on a blue stellar continuum. Its oxygen abundance, determined to be $7.34 \pm 0.09$, makes it one of the most metal-poor XBCDs discovered to date.

$\mathrm{J} 1044+0353$ : this object is likely a pair of physically associated SF regions with velocity and projected linear separation of $\sim 30 \mathrm{~km} \mathrm{~s}^{-1}$ and $0.8 \mathrm{kpc}$, respectively. The brighter, eastern SF region a displays extremely blue colours $(g-i \lesssim-1$ mag within a $3^{\prime \prime} \times 3^{\prime \prime}$ region), which are only reproducible by a photoionized region model involving strong nebular emission, in addition to a very young stellar population. Indeed, our spectra reveal nebular lines with an $E W$ of $318 \AA$ and $1420 \AA$ for the $\mathrm{H} \beta$ and [O III] $\lambda 5007$, respectively, implying an intense, ongoing SF episode. Our photometry indicates that more than $80 \%$ of the total emission in excess of the LSB host of J1044+0353 originates in region a.

In contrast, the faint eastern region $\mathrm{b}$, reflected on a weak bump in the SBPs at $R^{\star} \approx 2$ '.5, exhibits weak nebular emission with an $E W(\mathrm{H} \beta) \approx 20 \AA$ and a significantly redder colour of $g-i \approx-0.4 \mathrm{mag}$. From Fig. 6, we estimate the burst age of regions a and b to be $\$ 5 \mathrm{Myr}$ and $\sim 100 \mathrm{Myr}$, respectively. Both with regard to its absolute $g$ magnitude $(-15.9 \mathrm{mag})$ and exponential scale length $(\alpha=0.19 \mathrm{kpc})$, the LSB host of J1044+0353 is typical of BCDs. Its blue mean $g-i$ colour of $\sim 0$ mag strongly suggests a predominantly young stellar population with an age between 0.1 and 1 Gyr for SFH1 and SFH2, respectively. $\mathrm{J} 1044+0353$ therefore qualifies as a promising young XBCD candidate. On the other hand, it cannot be ruled out from the available data that the blue $g-i$ colours of its host are partly due to extended ionized gas emission associated with the SF region a.

Our derived oxygen abundance for region a, $12+\log (\mathrm{O} / \mathrm{H})=$ $7.44 \pm 0.01$, is close to the values of $7.48 \pm 0.01$ and $7.46 \pm$ 0.03 inferred from SDSS spectra by Kniazev et al. (2003) and Izotov et al. (2006a). For the fainter region b, not studied previously, we derive a comparably low oxygen abundance of $12+\log (\mathrm{O} / \mathrm{H})=7.45 \pm 0.09$.

$\mathrm{J} 1201+0211$ : star-forming activities in this system are confined to within a single compact $\left(R_{\mathrm{SF}}=0.14 \mathrm{kpc}\right)$ region at the southeastern tip of an elongated irregular LSB host. This source contributes less than $10 \%$ of the $g$ band emission of the galaxy and has properties characteristic of a very young stellar population $(g-i \approx-0.7 \mathrm{mag}, E W(\mathrm{H} \beta)=248 \AA)$. Our oxygen abundance determination for this HII region, $7.49 \pm 0.02$, is consistent with previous determinations based on SDSS spectra $(7.55 \pm 0.03$, Kniazev et al. 2003; $7.51 \pm 0.03$, Izotov et al. 2006a).

Image stacking reveals a chain of faint $\left(m_{g} \geq 26 \mathrm{mag}\right) \mathrm{com}-$ pact sources discernible out to $\sim 1.4 \mathrm{kpc}$ northwest of the HII region, most notably regions labeled nw1 and nw2 in Fig. 4c. The irregular host galaxy of $\mathrm{J} 1201+0211$ is relatively blue 
( $g-i=0.33 \pm 0.1$ for $R^{\star} \geq 4$.' 6 ), suggesting an age of between 1 and $\sim 2.3$ Gyr for SFH1 and SFH2, respectively.

J1414-0208: this system bears morphological resemblance to the XBCD 2dF 115901 (P06), however, in contrast to the latter, it shows mild SF activity. This is evident from its SBPs (Fig. 3), which do not display a central luminosity enhancement above the underlying host galaxy and by its relatively faint nebular emission $[E W(\mathrm{H} \beta)=48 \AA]$. Its irregular LSB host has a blue mean $g-i$ colour of $0.23 \pm 0.1 \mathrm{mag}$, which suggests a dominant stellar population with an age $<2$ Gyr (SFH2). This system has not been studied previously. Its oxygen abundance of $12+\log (\mathrm{O} / \mathrm{H})=7.28 \pm 0.32$, although relatively uncertain, places it within the XBCD metallicity range. From SDSS spectra, we derive a comparably low oxygen abundance of $12+\log (\mathrm{O} / \mathrm{H})=7.35 \pm 0.26$.

J2230-0006: with an exponential scale length of $\alpha=$ $0.17 \mathrm{kpc}, \mathrm{J} 2230-0006$ is the most compact galaxy in our sample. Its LSB host shows slight deviations from axis symmetry and a relatively blue $g-i$ colour of $\sim 0.3 \mathrm{mag}$. SF activities are mainly present in the southern part of this XBCD (cf. Fig. 4) and contribute about $20 \%$ of its total $g$ emission. The blue colour of its SF region $\left(-0.6 \mathrm{mag}\right.$ within a $4^{\prime \prime} \times 4^{\prime \prime}$ box $)$ and its amble nebular emission $[E W(\mathrm{H} \beta)=133 \AA]$ suggest a young starburst age. The oxygen abundance of J2230-0006 was determined to be $12+\log (\mathrm{O} / \mathrm{H})=7.64 \pm 0.02$, in good agreement with the value of $12+\log (\mathrm{O} / \mathrm{H})=7.66 \pm 0.04$ by Izotov et al. (2007b).

J2302+0049: this system contains two high surface brightness regions separated by 3 .' $4(\sim 2.2 \mathrm{kpc})$, which differ in their luminosity by a factor of approximately 5 . The brighter region a shows blue colours $(g-i=-0.78$ mag within a $2^{\prime \prime} \times 2^{\prime \prime}$ box $)$ and copious nebular emission $(E W(\mathrm{H} \beta)=212 \AA$, $E W([\mathrm{O}$ III $] \lambda 5007)=1145 \AA$ ) indicating a substantial population of hot massive stars. The oxygen abundance in this HII region was determined from the $3.6 \mathrm{~m}$ spectra to be $12+\log (\mathrm{O} / \mathrm{H})=$ $7.63 \pm 0.02$, close to the value of $12+\log (\mathrm{O} / \mathrm{H})=7.65 \pm$ 0.04 that we derive from SDSS spectra following Izotov et al. (2006a). The fainter region $\mathbf{b}$ shows no emission lines and the signal-to-noise ratio of the available spectra does not permit us to infer its redshift from stellar absorption lines.

The relatively large distance and intrinsic compactness of J2302+0049 prevent a detailed study of the properties of its host galaxy, which on the available images is marginally more extended than the SF component. If primarily due to stellar emission, its mean colour of $\sim 0.1 \mathrm{mag}$, is consistent with a young evolutionary status with an age between 0.25 and $1.4 \mathrm{Gyr}$ for SFH1 and SFH2, respectively.

\section{Discussion}

Including the seven galaxies studied here, the total number of $\mathrm{XBCDs}$ has increased to $\sim 35$. It is therefore timely to review their general photometric and morphological properties, in order to explore possible trends and better coordinate future searches for these systems in the nearby universe and at higher redshift.

A first important conclusion from the present and previous studies is that, similar to other dwarf galaxies, the host galaxy of XBCDs can be approximated by an exponential fitting law in its outer parts, i.e. for galactocentric radii $3 \alpha \lesssim$ $R^{\star} \lesssim 6 \alpha$. Additionally, several XBCDs display extended central flat cores in their host galaxies, reaching in some cases out to $R^{\star} \sim 3 \alpha$. Centrally flattened exponential profiles have also been observed in a sizeable fraction of early and latetype dwarfs spanning a wide range in absolute magnitude
(Binggeli \& Cameron 1991; Noeske et al. 2003, P96a). The XBCD - BCD connection is further supported by the fact that both object classes populate roughly the same locus in the $\mu_{\mathrm{E}, 0}$ versus $M_{\text {host }}$ and $\log (\alpha)$ versus $M_{\text {host }}$ parameter space (Fig. 8) and are comparable in their effective radius (P06).

These lines of evidence suggest that XBCDs do not represent peculiar cases of dwarf galaxy evolution, reflected in strongly distinct structural properties (e.g. an abnormally diffuse or an ultra-compact LSB host), but that they share similar structural properties and are therefore likely also to lie on a common evolutionary track with the main population of more metal-rich BCDs with $12+\log (\mathrm{O} / \mathrm{H}) \gtrsim 8$. Of course, this conclusion may not be free of selection biases, given that XBCDs are mostly detected by their high $E W(\mathrm{H} \beta)$, blue colours, and compactness. It is therefore conceivable that a substantial population of relatively quiescent and more diffuse metal-poor SF dwarfs remains strongly under-represented in current XBCD surveys. The irregular SF dwarf J0113+0052, discovered by Izotov et al. (2006b), may be regarded as an example of this kind. Additionally, it might be questioned that optical spectroscopic searches for XBCDs, based on forbidden line measurements in HII regions, can uncover systems with a gas-phase metallicity significantly lower than $12+\log (\mathrm{O} / \mathrm{H}) \sim 7.0\left(\sim Z_{\odot} / 60\right.$, adopting a solar abundance of 8.76, Caffau et al. 2008), thus better constrain the minimum level of chemical enrichment in the warm ISM of SF galaxies. This is because, below some abundance level and ionization parameter, oxygen forbidden lines become too weak to be measurable. For example, calculations with the photoionization code CLOUDY (Ferland et al. 1998) show that, at a metallicity $12+\log (\mathrm{O} / \mathrm{H})=7.0$, electron density $N_{\mathrm{e}}=100 \mathrm{~cm}^{-3}$ and ionization parameter $U=10^{-3}$, the oxygen [O III] $\lambda 4363$ and [O III] $] \lambda 5007$ line intensities decrease to $<1 \%$ and $38 \%$ of that of the $\mathrm{H} \beta$ line, respectively, a determination of the electron temperature based on the $[\mathrm{O}$ III $] \lambda 4363$ line becomes therefore practically impossible.

It is important to note that the detection of a stellar LSB host in all known XBCDs, including SBS 0335-052E (Izotov et al. 1997b; Thuan et al. 1997; P98) and I Zw 18 (Izotov et al. 2001; Papaderos et al. 2001; P02) suggests that none of these systems are currently forming in situ its first generation of stars. This is also indicated by spatially resolved evolutionary synthesis studies (Izotov et al. 1997b; Vanzi et al. 2000; Guseva et al. 2001, 2003c; Hunt et al. 2003) and colour-magnitude diagram analyses of selected systems (e.g., Izotov \& Thuan 2004b,c; Östlin \& Mouhcine 2005; Aloisi et al. 2007).

On the other hand, the existing work consistently suggests that the host galaxies of XBCDs are less evolved than those of BCDs. This is indicated by deep surface photometry studies that reveal uniformly blue colours in the XBCD hosts, with a $V-I$ and $g-i$ index in the range between $\sim 0.1$ and $\lesssim 0.5$ mag (e.g., P02; Guseva et al. 2003c, and references therein) and $<0.4$ mag (this paper), respectively. By contrast, the LSB hosts of $\mathrm{iE} / \mathrm{nE} \mathrm{BCDs}$, representing $\sim 90 \%$ of the BCD population (LT86) show typically red ( $\sim 1 \mathrm{mag}) B-R$ and $V-I$ colours (P96b; Cairós et al. 2001b; Gil de Paz \& Madore 2005).

Another important, largely overlooked aspect concerns the morphology of XBCDs. The hosts of these systems reveal in their majority conspicuous deviations from axis-symmetry suggesting a little degree of dynamical relaxation. By this, they again significantly differ from the bona fide old, more metal-rich $\mathrm{iE} / \mathrm{nE}$ BCDs whose defining property is a smooth elliptical LSB host galaxy.

Faint, non-axisymmetric distortions are present in roughly one half of the XBCDs studied here. The irregular outer 

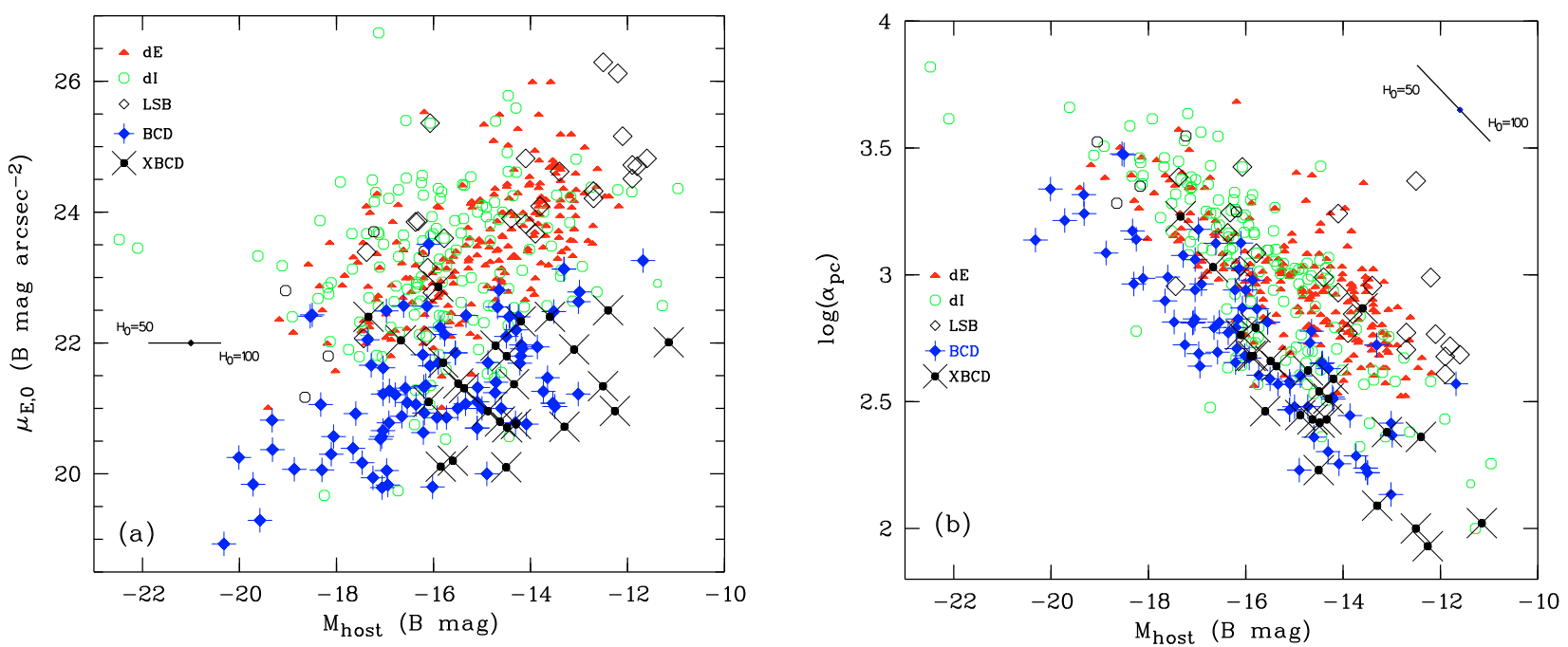

Fig. 8. Comparison of the structural properties of the host galaxy of XBCDs, BCDs, dIs, dwarf ellipticals (dEs) and Low-Surface Brightness (LSB) galaxies. Data for iE/nE BCDs are compiled from Cairós et al. (2001a), Drinkwater \& Hardy (1991), Marlowe et al. (1997), Papaderos \& Fricke (1998), Noeske et al. (2000), P96a, Papaderos (1998) and P02. Data for other types of dwarf galaxies are taken from Binggeli \& Cameron (1991), Binggeli \& Cameron (1993), Bothun et al. (1991), Caldwell \& Bothun (1987), Carignan \& Beaulieu (1989), Hopp \& Schulte-Ladbeck (1991), Patterson \& Thuan (1996), Vigroux et al. (1986) and van Zee (2000). Photometric quantities in the SDSS $g$ and $V$ band were transformed into $B$ band assuming $B-g=0.3 \mathrm{mag}$ and $B-V=0.5 \mathrm{mag}$ (see discussion in Sect. 3.2 .2 and in P06). The lines show the shift of the data points caused by a change of the Hubble constant from 75 to 50 and $100 \mathrm{~km} \mathrm{~s}^{-1} \mathrm{Mpc}^{-1}$. a) Central surface brightness $\mu_{\mathrm{E}, 0}$ vs. absolute $B$ magnitude $M_{\text {host }}$ of the LSB component. b) Logarithm of the exponential scale length $\alpha$ in pc vs. $M_{\text {host }}$. Data for XBCDs are compiled from Papaderos et al. (1998), Papaderos et al. (1999), Kniazev et al. (2000), Guseva et al. (2001), Fricke et al. (2001), P02, Guseva et al. (2003a), Guseva et al. (2003b), Guseva et al. (2003c), P06 and Pustilnik et al. (2005). Five further systems with an oxygen abundance slightly above $12+\log (\mathrm{O} / \mathrm{H})=7.6$ are also included: 2dF 169299, UM 570, UM 559 and $2 \mathrm{dF} 84585(12+\log (\mathrm{O} / \mathrm{H})=7.68,7.71,7.72,7.66$, respectively; P06 $)$ and $\operatorname{Pox} 186(12+\log (\mathrm{O} / \mathrm{H})=7.74$, Guseva et al. 2004).

morphology of those systems is certainly not due to insufficient detection of their underlying galaxy host. This is because, as is evident from Fig. 4, SDSS images allow us to interpolate contours down to a $g$ band surface brightness $\mu_{g} \geq 25.5 \mathrm{mag} / \square^{\prime \prime}$, corresponding to $\mu_{B} \approx 25.8 \mathrm{mag} / \square^{\prime \prime}$. At such intensity levels the elliptical host of evolved BCDs dominates the light (P96b; Cairós et al. 2001b; P02) and should have been detected if it were present. Furthermore, with the possible exception of J1201+0211, stacked gri images do not reveal tidal features in any of our sample SDSS galaxies, ruling out strong gravitational interactions or galaxy merging as the origin of the observed morphological distortions. Widespread SF activity in the LSB host can also be excluded from $g-i$ colour maps and long-slit spectra.

Likewise, most of the XBCDs investigated previously on the basis of deep surface photometry are as well characterised by irregular host galaxies. Such examples are SBS 0335-052W (12+ $\log (\mathrm{O} / \mathrm{H})=7.12$, Izotov et al. 2005; see P98 and Papaderos et al. $2006 \mathrm{c}$, for photometry), Tol $65(12+\log (\mathrm{O} / \mathrm{H})=7.54$, Izotov et al. 2004a; see Papaderos et al. 1999, for photometry), SBS $1415+437(12+\log (\mathrm{O} / \mathrm{H})=7.6$, Thuan et al. 1999; Guseva et al. 2003c), Tol 1214-277 $(12+\log (\mathrm{O} / \mathrm{H})=7.55$, Izotov et al. 2004a; see Fricke et al. 2001, for photometry), I Zw $18(12+\log (\mathrm{O} / \mathrm{H})=$ 7.17, Izotov et al. 1997a; see Papaderos et al. 2001; and P02 for photometry), SBS $0940+544(12+\log (\mathrm{O} / \mathrm{H})=7.46$, Guseva et al. 2001), SBS $1129+576(12+\log (\mathrm{O} / \mathrm{H})=7.36$, Guseva et al. 2003a), HS $0837+4717(12+\log (\mathrm{O} / \mathrm{H})=7.64$, Pustilnik et al. 2004b), DDO $68(12+\log (\mathrm{O} / \mathrm{H})=7.21 \ldots 7.13$, Pustilnik et al. 2005; Izotov \& Thuan 2007, respectively), HS 2134+0400 $(12+\log (\mathrm{O} / \mathrm{H})=7.44$, Pustilnik et al. 2006; Guseva et al. 2007), J2104-0035 and $\mathrm{J} 0113+0052(12+\log (\mathrm{O} / \mathrm{H}) \approx 7.2$ and 7.26, respectively, Izotov et al. 2006b), 2dF 171716, 2dF 115901 $(12+\log (\mathrm{O} / \mathrm{H})=7.5,7.57$, respectively; P06) and J0301-0052,
$\mathrm{J} 0911+3135, \mathrm{~J} 2238+1400(12+\log (\mathrm{O} / \mathrm{H})=7.52,7.51$ and 7.56 , respectively, Izotov \& Thuan 2007). This is also the case for several systems with an oxygen abundance slightly above $12+\log (\mathrm{O} / \mathrm{H})=7.6$, such as $2 \mathrm{dF} 84585$ and $2 \mathrm{dF} 169299$ $(12+\log (\mathrm{O} / \mathrm{H})=7.66$ and 7.68 , respectively; P06).

With regard to the morphological properties of the starforming component of XBCDs (e.g. multiplicity, luminosity distribution, and the degree of the confinement of SF regions to the center of the XBCD host), these have never been studied in a systematic manner before, and a comparison with $\mathrm{iE} / \mathrm{nE}$ BCDs would therefore be premature at this point. However, two trends are apparent. First, in a significant fraction of XBCDs, star-forming activities are not strongly confined to the geometrical center of the host galaxy and, second, the global SF process in several of these systems appears to be largely driven by propagation.

Figure 9 contains several examples of XBCDs with offcenter SF activity, including $2 \mathrm{dF}$ 115901, SBS 0335-052W, J1414-0208, and J0911+3135. The most impressive instances of off-center SF activity among XBCDs are cometary systems (iI,C type in the BCD classification scheme devised by LT86). These objects contain a luminous SF region at the one tip of an elongated blue and irregular host galaxy with a gradually decreasing surface brightness towards its antipodal end. The hypothesis that these systems are edge-on disks with a dominant SF complex in their outermost periphery can be dismissed on statistical grounds. Some iI,C XBCDs (for example, SBS $1415+437$ and SBS $0940+544$ ) display signatures of low-level ongoing or recent star formation along their major axis or colour gradients suggesting propagation of SF activities from the farend side of their elongated host galaxy towards the young, dominant SF region (see e.g. P98; Guseva et al. 2001, 2003c). 
That the formation of the stellar component in a XBCD may largely be driven by SF propagation has been suggested from an analysis of SBS 0335-052E $(12+\log (\mathrm{O} / \mathrm{H})=7.3 \ldots 7.2$, Izotov et al. 1997b; Papaderos et al. 2006a, respectively), for which P98 have estimated a SF propagation velocity of $\sim 20 \mathrm{~km} \mathrm{~s}^{-1}$, of the order of the sound speed in the warm ISM. It is unclear for how long this process can continue; however, as long as gas supply of the appropriate temperature and density is available ahead of the SF front, no self-limiting mechanism should exist. Propagating star formation with a constant speed $u$ over a period $\tau$ may naturally lead to a cometary morphology on a linear scale $l \sim 10 \mathrm{kpc} \times\left(u / 10 \mathrm{~km} \mathrm{~s}^{-1}\right) \times(\tau / 1 \mathrm{Gyr})$. As evident from Fig. 9, $l$ is of the order of the projected major-axis of several cometary XBCDs.

We note that the iI,C morphology is not uniquely observed in $\mathrm{XBCDs}$ and that examples of this kind also exist among BCDs of higher metallicity (Noeske et al. 2000; Cairós et al. 2001a; Noeske et al. 2003; Gil de Paz et al. 2003). The essential trend, however, is that whereas systems with cometary morphology or strongly off-center SF activity comprise less than $10 \%$ of the BCD population (LT86), they apparently dominate the XBCD population. In this context, it is worth pointing out that studies of iI,C BCDs in the metallicity range between $\approx 7.8$ and 8.0 by Noeske et al. (2000) suggest that these systems are younger ( $\$ 4 \mathrm{Gyr}$ ) than the main class of $\mathrm{iE} / \mathrm{nE} \mathrm{BCDs}$, they are therefore likely to represent intermediate stages of BCD evolution. This conjecture is consistent with the high incidence of cometary systems among young XBCD candidates discussed here.

It is unclear whether or not cometary morphology is linked to galaxy interactions, as neither observations nor numerical simulations presently provide tight constraints in this respect. However, it is known that a significant fraction ( $>30 \%)$ of BCDs are not truly isolated but have optically faint nearby companions (Noeske et al. 2001; Pustilnik et al. 2001). Dwarf galaxy encounters with a wide range of impact parameters are therefore likely to play an important role in BCD evolution. The frequency of strong collisions and, eventually, subsequent merging of BCD progenitors is still not constrained well. However, several notable examples (iI,M BCDs in the classification scheme of LT86) exist in the samples of Cairós et al. (2001a) and Gil de Paz et al. (2003), and there is growing evidence that the major fraction of intrinsically luminous $\left(M_{B}<-18\right.$ mag) Blue Compact Galaxies are of merger origin (Östlin et al. 2001). In addition, the morphology of some XBCDs (for example, 2dF 169299, $2 \mathrm{dF}$ 115901, and 2dF 171716; cf. Fig. 9) is consistent with the merger interpretation. The importance of gravitational interactions to BCD evolution is indicated further by radio interferometry that reveals HI clouds in the close vicinity ( $\$ 100 \mathrm{kpc})$ of numerous systems (Taylor et al. 1993; Thuan et al. 2004).

The hypothesis that strong gravitational interactions or galaxy merging are the origin of cometary morphology does not however appear to be tenable. If cometary BCDs were indeed forming by galaxy merging, one would expect tidal features to protrude far beyond their Holmberg radius and be readily detectable at surface brightness levels of $\mu \sim 24 \mathrm{~B}$ mag/ $\square^{\prime \prime}$, in a similar way to merging disk galaxies. Since the visibility timescale of tidally ejected stellar and gaseous matter is long ( $\sim 1$ Gyr; Hibbard \& Mihos 1995, and references therein), of the order of the luminosity-weighted age of the XBCD galaxy host, these features should be almost ubiquitous in iI,C systems. The probability that both merging counterparts are metal poor and retain their gas-phase metallicity of a level $12+\log (\mathrm{O} / \mathrm{H}) \lessgtr 7.6$, even after a strong, merging-induced starburst, is also low.
A more viable interpretation involves weak interactions with low-mass stellar or gaseous companions. These may have a twofold effect, leading to a bar-like gas distribution and triggering SF activities that, by propagation along the direction of maximum gas density, could subsequently produce a cometary BCD morphology. Alternatively, a propagating shock wave induced by gas-cloud infall onto a quiescent late-type dwarf might generate a similar star formation pattern.

In summary, the hypothesis that SF propagation is the main process driving the formation of cometary XBCDs is not in conflict with the hypothesis that the evolution of these systems is largely influenced by interactions. However, lacking theoretical guidence and robust statistics on the gas distribution and kinematics of these systems, the possible role of interactions cannot be reliably assessed. However, numerical simulations of increasing sophistication continue to reproduce important properties of SF dwarfs (e.g. Noguchi 2001; Recchi et al. 2002; Pelupessy et al. 2004, 2006; Hensler et al. 2004; Bekki 2008), and promise to provide key insights into the star formation history and morphological evolution of cometary dwarfs in the near future. Dedicated observational studies of these extremely metal-poor cometary galaxies will also hopefully provide important constraints and incentives for theoretical work.

As mentioned above, a compelling argument that XBCDs have undergone previous evolution derives from the detection of an extended stellar host galaxy. The blue, luminosity-weighted colours of this component, interpreted in the framework of simple SFH parametrisations (e.g., SFH2) suggest that XBCDs form a heterogeneous class of predominantly cosmologically young objects, with examples among them which have formed most of their stellar mass in the past few $10^{8}$ yrs and are possibly still experiencing the major phase of their dynamical assembly (e.g. SBS 0335-052E, I Zw 18) to moderately evolved cometary systems that likely formed most of their stellar mass within the last $\sim 2$ Gyr (Tol 65, Tol 1214-277, SBS 1415+437, SBS 0940+544, $\mathrm{J} 1044+0353)$. This conclusion is supported further by detailed evolutionary synthesis models (e.g. Izotov et al. 2001; Guseva et al. 2001, 2003a,c) involving more complex SFHs and which aim to account self-consistently for a variety of observables, such as the EWs of Balmer emission and absorption lines, the slope of the spectral energy distribution (SED), and spatially resolved colours.

In the case of the XBCD I Zw 18, CMD studies based on HST ACS images do not converge into a broadly accepted interpretation about the age of its stellar component, yielding values between $\leq 0.5$ Gyr (Izotov \& Thuan 2004b) and >1 Gyr (Aloisi et al. 2007). This might be partly due to the specific properties of this system. Papaderos et al. (2002) showed that the exponential LSB host of I Zw 18, which was previously thought to be dominated by stellar emission, is due entirely to extended, patchy ionized gas emission, therefore extreme caution is required when using CMDs and surface photometry to place constraints on the formation history of its stellar component. The stellar component of I Zw 18 is by a factor of approximately 2 more compact that the ionized gas halo and, in contrast to the main class of evolved BCDs, has uniformly blue colours down to a surface brightness level of $\mu \sim 26 \mathrm{Bmag} / \square^{\prime \prime}$. Additionally, as found by Cannon et al. (2002), I Zw 18 shows a highly inhomogeneous extinction pattern, a fact that further complicates CMD analyses. The nature of point sources detected in the presense of dominant ionized gas emission in I Zw 18 , at a distance of $18.2 \mathrm{Mpc}$ (Aloisi et al. 2007), which corresponds to a projected area of $\sim 20 \mathrm{pc}^{2}$ per HST ACS pixel, is undoubtedly an important question in XBCD research. 


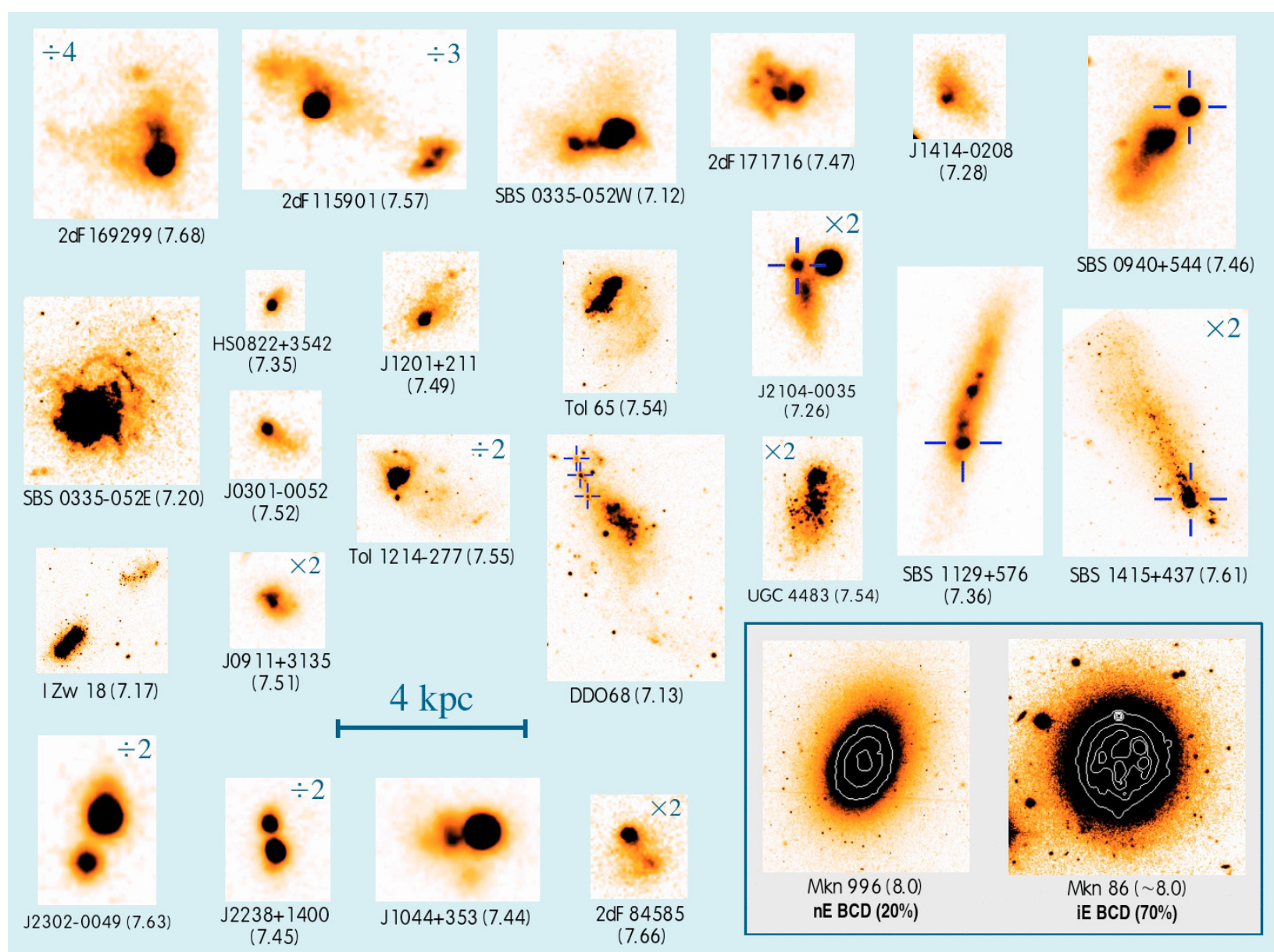

Fig. 9. Comparison of systems in the range of XBCD metallicity with the main class of the more metal-rich, bona fide old BCDs Mkn 996 and Mkn 86. These two systems may be regarded as prototypical examples of the dominant nE/iE morphological BCD type (cf. LT86). All galaxy images were scaled to a common distance to better facilitate a comparison. Those images that are displayed magnified or downsized for the sake of better visibility are labeled with the respective scaling factor. Off-center low-metallicity HII regions are marked with crosses for clarity. Within brackets we indicate the oxygen abundance of the lowest-metallicity HII region in each XBCD. Metallicities are taken from the literature sources given in pages 125 and 126. The images are from P06 (2dF 169299, 2dF 115901, 2dF 171718, 2dF 84585), P98 (SBS 0335-052E, see also Thuan et al. 1997, HST program 5408), Papaderos et al. (2006c, SBS 0335-052W), Guseva et al. (2001, SBS 0940+544), Guseva et al. (2003a, SBS 1129+576), Guseva et al. (2003c, SBS 1415+437, HST program 5408), Papaderos et al. (2001, I Zw 18, $R$ band HST WFPC2 exposure with the H $\alpha$ emission subtracted out, HST program 5309), the HST archive (Tol 65, Tol 1214-277, Mkn 996; HST programs 5408 and 6678), Papaderos \& Noeske (2008, in preparation, HS 0822+3542, UGC 4483, Mkn 86), and from the SDSS (J1414-0208, J1044+353, J2302-0049, J1201+211, J2104-0035, J0301-0052, J0911+3135, DDO68, J2238+1400).

The formation history of the XBCD host is clearly a crucial and outstanding issue. XBCDs may all contain a faint substrate of stars of cosmological age (see discussion in P98), quite similar to the ancient metal-poor stellar population in Local Group dwarf spheroidals (Grebel \& Gallagher 2004). As pointed out in P98, this putative ancient stellar background cannot be entirely ruled out on spectrophotometric grounds, since its effect on the observed SED might be barely detectable. It is only possible to infer an upper bound to the mass fraction of this hypothetical old stellar population that, by necessity, is tied to simplifying assumptions about the SFH and intrinsic extinction. Current upper limits for a few thoroughly analysed XBCDs range between $\sim 15 \%$ and $<50 \%$ (Vanzi et al. 2000; Guseva et al. 2001, 2003c; Hunt et al. 2003; Pustilnik et al. 2004a).

Arguably, a low mass fraction of ancient stars possibly present in XBCDs do not rule out the young evolutionary status of these systems. This would only be the case if the formation epoch of those first stars were coeval with the dominant phase of XBCD formation, and XBCDs/BCDs were invariably forming in a single, short (1-3 Gyr) SF episode that converted most of their gaseous reservoir into stars. This interpretation is untenable, however, inter alia, because of the gasrichness and recurrent starburst activity of these systems (see e.g. Thuan 1991; Mas-Hesse \& Kunth 1999). As a matter of fact, dwarf galaxies of a given baryonic mass may have followed quite diverse evolutionary pathways. This is manifestated in e.g. the Local Group galaxies for which we observe an impressive manyfold of SFHs and morphologies, comprising both ancient metal-poor dwarf spheroidals and metal-rich dEs (e.g. NGC 185, NGC 205 Dolphin et al. 2005, see also Mateo 1998) as well as late-type irregulars that underwent the dominant phase of their build-up only 1 Gyr ago (e.g. Sextans A, Dolphin et al. 2003). The inherent variety of SFHs in dwarf galaxies is further enhanced by the role of the environment, which is recognised to be an important factor in galaxy evolution (Butcher \& Oemler 1984; Dressler 1980; Dressler et al. 1997; Poggianti et al. 1997; Pustilnik et al. 2001). For example, it is well established that the $E W(\mathrm{H} \beta)$ of SF dwarfs increases towards the periphery of galaxy clusters (Vílchez 1995) and that XBCDs preferentially populate theextreme field (Pustilnik et al. 2001). These findings are 
consistent with the idea that the formation timescale of relatively isolated late-type dwarfs is the longest, making low-density regions promising sites to search for young XBCDs candidates. In view of such considerations, the existence of a small number of unevolved XBCDs in the nearby universe is unsurprising, and a manifestation of the diversity in the SFHs of dwarf galaxies.

\section{Summary}

We have presented spectroscopic observations with the $3.6 \mathrm{~m}$ ESO telescope of eight H II regions in seven emission-line galaxies, selected from the Data Release 4 (DR4) of the Sloan Digital Sky Survey (SDSS) (six galaxies) and from the Six-Degree Field Galaxy Redshift Survey (6dFGRS) (one galaxy). The three brightest of these sources were first identified by Kniazev et al. (2003, 2004) using SDSS data and their oxygen abundances were measured using SDSS spectra.

From our $3.6 \mathrm{~m}$ long-slit data we determined the oxygen abundance of these systems to be $12+\log (\mathrm{O} / \mathrm{H}) \lesssim 7.6$, which places them among the most metal-poor star-forming (SF) galaxies ever discovered. Furthermore, we used imaging data to study the morphology and surface brightness and colour distribution of the SDSS galaxies in our sample. From decomposition of surface brightness profiles (SBPs), we infered a wide range of between $\sim 5 \%$ and $\sim 50 \%$ for the luminosity contribution of the $\mathrm{SF}$ component to the total $g$ band emission. We also measured a wide range of equivalent widths for the strong nebular lines, varying between $\sim 20 \AA$ and $\sim 320 \AA$ for the $\mathrm{H} \beta$ line and between $\sim 40 \AA$ and $\sim 1400 \AA$ for the [OIII] $\lambda 5007$ line. The effect of copious ionized gas emission manifests itself also in extraordinarily blue colours $(g-i \lesssim-0.8 \mathrm{mag})$ in the SF component of several galaxies studied.

The present investigation of extremely metal-poor SF galaxies (hereafter XBCDs) in conjunction with data from the literature leads to the following conclusions:

i) All XBCDs known possess a compact to moderately extended stellar host galaxy underlying their SF component, thus they are not experiencing the in situ formation of their first stellar generation in a galaxy-wide starburst.

ii) $\mathrm{XBCDs}$ are with respect to the exponential outer slope and the structural properties of their host galaxies fairly comparable to the main type $(\sim 90 \%)$ of the bona fide old and more metal-rich $\mathrm{iE} / \mathrm{nE}$ blue compact dwarf (BCD) galaxies. This suggests that XBCDs do not represent peculiar cases of dwarf galaxy evolution, reflected by their distinctive structural properties (e.g. abnormally diffuse or ultra-compact host galaxies), but that they probably follow a common evolutionary track with the main population of BCDs.

iii) The host galaxies of XBCDs are substantially bluer than the hosts of $\mathrm{iE} / \mathrm{nE} \mathrm{BCDs}$, suggesting that the dominant mass fraction of the stellar component in these systems formed within the past $\sim 2$ Gyr. The possibility that XBCDs contain a low mass fraction of old $(\sim 10 \mathrm{Gyr})$ stars cannot be dismissed.

iv) The host galaxies of XBCDs typically show conspicuous morphological distortions in their periphery, suggesting a little degree of dynamical relaxation. In this property, they again markedly differ from the main class of the old, elliptical BCDs. XBCDs are also different from typical BCDs in terms of their lower spatial confinement of SF activities to their geometrical center. Most notably, a large fraction of XBCDs show a cometary morphology, characterised by the presence of a dominant star-forming region at one tip of an elongated, blue stellar host. Surface brightness and colour gradients along that host galaxy can be plausibly accounted for by propagation of star-forming activities along its body.

The overall conclusion emerging from the present study is that XBCDs form a heterogeneous class of predominantly cosmologically young objects, with examples among them which are still undergoing the major phase of their formation, to moderately evolved cometary systems that have formed the dominant mass fraction of their stellar component within the past $\sim 2 \mathrm{Gyr}$. $\mathrm{XBCDs}$ may therefore hold important insights into the early morphological and dynamical evolution of low-mass protogalaxies in the distant universe.

Acknowledgements. P.P. would like to thank Prof. A. Zensus and the MaxPlanck-Institute for Radioastronomy in Bonn for their hospitality. N.G.G. and Y.I.I. thank the hospitality of the Institute for Astrophysics (Göttingen) and the support of the DFG grant No. 436 UKR 17/15/06. P.P. would like to thank Gaspare Lo Curto, Lorenzo Monaco, Carlos La Fuente, Eduardo Matamoros and the whole ESO staff at the La Silla Observatory for their support. Y.I.I. acknowledges the partial financial support of NSF grant AST 02-05785. All the authors acknowledge the work of the Sloan Digital Sky Survey (SDSS) team. Funding for the SDSS has been provided by the Alfred P. Sloan Foundation, the Participating Institutions, the National Aeronautics and Space Administration, the National Science Foundation, the US Department of Energy, the Japanese Monbukagakusho, and the Max Planck Society. The SDSS Web site is http:// www.sdss.org/. We would like to thank the Anglo-Australian Observatory staff at the UK Schmidt Telescope and the entire 6dFGS team for ensuring the success of the 6dFGS. This research has made use of the NASA/IPAC Extragalactic Database (NED) which is operated by the Jet Propulsion Laboratory, CALTECH, under contract with the National Aeronautic and Space Administration. This publication makes use of data products from the Two Micron All Sky Survey, which is a joint project of the University of Massachusetts and the Infrared Processing and Analysis Center/California Institute of Technology, funded by the National Aeronautics and Space Administration and the National Science Foundation. We are grateful to the anonymous referee for her/his helpful comments and suggestions.

\section{References}

Adelman-McCarthy, J. K., Agüeros, M. A., Allam, S. S., et al. 2006, ApJS, 162, 38

Aloisi, A., Clementini, G., Tosi, M., et al. 2007, ApJ, 667, L151

Barton, E. J., \& van Zee, L. 2001, ApJ, 550, L35

Bekki, K. 2008, MNRAS, in press [arXiv:0805.0662v1]

Binggeli, B., \& Cameron, L. M. 1991, A\&A, 252, 27

Binggeli, B., \& Cameron, L. M. 1993, A\&AS, 98, 297

Bothun, G. D., Impey, C. D., \& Malin, D. F. 1991, ApJ, 376, 404

Böhringer, H., \& Hensler, G. 1989, A\&A, 215, 147

Brinchman, J., Kunth, D., \& Durret, F. 2008, A\&A, 485, 657

Butcher, H., \& Oemler, A. 1984, ApJ, 285, 426

Caffau, E., Ludwig, H.-G., Steffen, M., et al. 2008 [arXiv: 0805.4398v1]

Cairós, L. M., Vílchez, J. M., González Pérez, J. N., Iglesias-Páramo, J., \& Caon, N. 2001a, ApJS, 133, 321

Cairós, L. M., Vílchez, J. M., González Pérez, J. N., Iglesias-Páramo, J., \& Muñoz-Tuñón, C. 2001b, ApJS, 136, 393

Cairós, L. M., Caon, N., Papaderos, P., et al. 2003, ApJ, 593, 312

Caldwell, N., \& Bothun, G. D. 1987, AJ, 94, 1126

Cannon, J. M., Skillman, E. D., Garnett, D. R., \& Dufour, R. J. 2002, ApJ, 565, 931

Carignan, C., \& Beaulieu, S. 1989, ApJ, 347, 760

Crowther, P. A., \& Hadfield, L. J. 2006, A\&A, 449, 711

Dekel, A., \& Woo, J. 2003, MNRAS, 344, 1131

Dolphin, A. E., Saha, A., Skillman, E. D., et al. 2003, AJ, 126, 187

Dolphin, A. E., Weisz, D. R., Skillman, E. D., \& Holtzman, J. A. 2005, in

Resolved Stellar Populations, Cancun, Mexico

[arXiv: astro-ph/0506430v1]

Dressler, A. 1980, ApJ, 236, 351

Dressler, A., Oemler, A., Jr., Couch, W. J., et al. 1997, ApJ, 490, 577

Drinkwater, M., \& Hardy, E. 1991, AJ, 101, 94

Ferland, G. J., Korista, K. T., Verner, D. A., et al. 1998, PASP, 110, 761

Fioc, M., \& Rocca-Volmerange, B. 1997, A\&A, 326, 950

Fricke, K. J., Izotov, Y. I., Papaderos, P., Guseva, N. G., \& Thuan, T. X. 2001,

AJ, 121, 169

Gil de Paz, A., \& Madore, B. F. 2005, ApJS, 156, 345 
Gil de Paz, A., Madore, B. F, \& Pevunova, O. 2003, ApJS, 147, 29

Grebel, E. K., \& Gallagher, J. S., III 2004, ApJ, 610, L89

Guseva, N. G., Izotov, Y. I., \& Thuan, T. X. 2000, ApJ, 531, 776

Guseva, N. G., Izotov, Y. I., Papaderos, P., et al. 2001, A\&A, 378, 756

Guseva, N. G., Papaderos, P., Izotov, Y. I., et al. 2003a, A\&A, 407, 74

Guseva, N. G., Papaderos, P., Izotov, Y. I., et al. 2003b, A\&A, 407, 91

Guseva, N. G., Papaderos, P., Izotov, Y. I., et al. 2003c, A\&A, 407, 105

Guseva, N. G., Papaderos, P., Izotov, Y. I., Noeske, K. G., \& Fricke, K. J. 2004,

A\&A, 421, 519

Guseva, N. G., Izotov, Y. I., Papaderos, P., \& Fricke, K. J. 2007, A\&A, 464, 885

Heckman, T. M., Dahlem, M., Lehnert, M. D., et al. 1995, ApJ, 448, 98

Hensler, G., Theis, C., \& Gallagher, J. S. III 2004, A\&A, 426, 25

Hibbard, J. E., \& Mihos, J. C. 1995, AJ, 110, 140

Hopp, U., \& Schulte-Ladbeck, R. E. 1991, A\&A, 248, 1

Hunt, L. K., Thuan, T. X., \& Izotov, Y. I. 2003, ApJ, 588, 281

Izotov, Y. I., \& Thuan, T. X. 1998a, ApJ, 497, 227

Izotov, Y. I., \& Thuan, T. X. 1998b, ApJ, 500, 188

Izotov, Y. I., \& Thuan, T. X. 1999, ApJ, 511, 639

Izotov, Y. I., \& Thuan, T. X. 2004a, ApJ, 602, 200

Izotov, Y. I., \& Thuan, T. X. 2004b, ApJ, 616, 768

Izotov, Y. I., \& Thuan, T. X. 2004c, ApJ, 567, 875

Izotov, Y. I., \& Thuan, T. X. 2007, ApJ, 665, 1115

Izotov, Y. I., Foltz, C. B., Green, R. F., Guseva, N. G., \& Thuan, T. X. 1997a, ApJ, 487, L37

Izotov, Y. I., Lipovetsky, V. A., Chaffee, F. H., et al. 1997b, ApJ, 476, 698

Izotov, Y. I., Chaffee, F. H., Foltz, C. B., et al. 1999, ApJ, 527, 757

Izotov, Y. I., Chaffee, F. H., Foltz, C. B., et al. 2001, ApJ, 560, 222

Izotov, Y. I., Papaderos, P., Guseva, N. G., Fricke, K. J., \& Thuan, T. X. 2004a, A\&A, 421, 539

Izotov, Y. I., Stasińska, G., Guseva, N. G., \& Thuan, T. X. 2004b, A\&A, 632, 210

Izotov, Y. I., Thuan, T. X., \& Guseva, N. G. 2005, ApJ, 415, 87

Izotov, Y. I., Stasińska, G., Meynet, G., Guseva, N. G., \& Thuan, T. X. 2006a, A\&A, 448, 955

Izotov, Y. I., Papaderos, P., Guseva, N. G., Fricke, K. J., \& Thuan, T. X. 2006b, A\&A, 454, 137

Izotov, Y. I., Thuan, T. X., \& Stasińska, G. 2007a, ApJ, 662, 15

Izotov, Y. I., Thuan, T. X., \& Guseva, N. G. 2007b, ApJ, 671, 1297

Jones, D. H., Saunders, W., Read, M., \& Colless, M. 2005, PASA, 22, 277

Kakazu, Y., Cowie, L. L., \& Hu, E. M. 2007, ApJ, 668, 853

Kewley, L. J., Brown, W. R., Geller, M. J., Kenyon, S. J., \& Kurtz, M. J. 2007, AJ, 133, 882

Kniazev, A. Y., Pustilnik, S. A., Masegosa, J., et al. 2000, A\&A, 357, 101

Kniazev, A. Y., Grebel, E. K., Hao, L., et al. 2003, ApJ, 593, 73

Kniazev, A. Y., Pustilnik, S. A., Grebel, E. K., Lee, H., \& Pramskij, A. G. 2004, ApJS, 153, 429

Kraan-Korteweg, R. C. 1986, A\&AS, 66, 255

Kudritzki, R. P. 2002, ApJ, 577, 389

Kunth, D., \& Sargent, W. L. W. 1983, ApJ, 273, 81

Kunth, D., \& Östlin, G. 2000, ARA\&A, 10, 1

Lee, H., Skillman, E. D., Cannon, J. M., et al. 2006, ApJ, 647, 970

Leitherer, C., Robert, C., \& Drissen, L. 1992, ApJ, 401, 596

Loose, H.-H., \& Thuan, T. X. 1986, in Star-Forming Galaxies and Related Objects, ed. D. Kunth, T. X. Thuan, \& T. T. Van (Paris: Editions Frontières), 73 (LT86)

Makarova, L. N., \& Karachentsev, I. D. 1998, A\&AS, 133, 181

Marlowe, A. T., Meurer, G. R., Heckman, T. M., \& Schommer, R. 1997, ApJS, 112,285

Martin, C. L., Kobulnicky, H. A., \& Heckman, T. M. 2002, ApJ, 574, 663

Mas-Hesse, J. M., \& Kunth, D. 1999, 349, 765

Mateo, M. 1998, ARA\&A, 36, 435

Noeske, K. G., Guseva, N. G., Fricke, K. J., et al. 2000, A\&A, 361, 33

Noeske, K. G., Iglesias-Páramo, J., Vílchez, J. M., Papaderos, P., \& Fricke, K. J. 2001, A\&A, 371, 806

Noeske, K. G., Papaderos, P., Cairós, L. M., \& Fricke, K. J. 2003, A\&A, 410, 481

Noeske, K. G., Papaderos, P., Cairós, L. M., \& Fricke, K. J. 2005, A\&A, 429, 115

Noeske, K. G., Koo, D. C., Phillips, A. C., et al. 2006, ApJ, 640, L143

Noguchi, M. 2001, ApJ, 555, 289
Östlin, G., \& Mouhcine, M. 2005, A\&A, 433, 797

Östlin, G., Amram, P., Bergvall, N., et al. 2001, A\&A, 374, 800

Ott, J., Walter, F., \& Brinks, E. 2005, MNRAS, 358, 1423

Papaderos, P. 1998, Ph.D. Thesis, University of Göttingen

Papaderos, P., \& Fricke, K. J. 1998, in X-rays from Blue Compact Dwarf Galaxies, in Highlights in X-ray astronomy: international symposium in honour of Joachim Trümper's 65th birthday, June 1998, Garching, ed. B. Aschenbach, \& M. Freyberg, MPE Rep., 272, 193

Papaderos, P., Loose, H.-H., Thuan, T. X., \& Fricke, K. J. 1996a, A\&AS, 120, 207 (P96a)

Papaderos, P., Loose, H.-H., Fricke, K. J., \& Thuan, T. X. 1996b, A\&A, 314, 59 (P96b)

Papaderos, P., Izotov, Y. I., Guseva, N. G., Thuan, T. X., \& Fricke, K. J. 1998, A\&A, 338, 43 (P98)

Papaderos, P., Fricke, K. J., Thuan, T. X., Izotov, Y. I., \& Nicklas, H. 1999, A\&A, 352, L57

Papaderos, P., Izotov, Y. I., Noeske, K. G., Thuan, T. X., \& Fricke, K. J. 2001, in Dwarf Galaxies and their Environment, ed. K. S. de Boer, R. J. Dettmar, \& U. Klein (Shaker Verlag), 111

Papaderos, P., Izotov, Y. I., Thuan, T. X., et al. 2002, A\&A, 393, 461 (P02)

Papaderos, P., Izotov, Y. I., Guseva, N. G., Thuan, T. X., \& Fricke, K. J. 2006a, A\&A, 454, 119

Papaderos, P., Izotov, Y. I., Guseva, N. G., et al. 2006b, A\&A, 457, 45

Papaderos, P., Yakobchuk, T., Izotov, Y. I., Guseva, N. G., \& Fricke, K. J. 2006c, in Galaxy Evolution Across the Hubble Time, ed. F. Combes, \& J. Palous (Prague: Cambridge University Press), IAU Symp., \#235, 326

Patterson, R. J., \& Thuan, T. X. 1996, ApJS, 107, 103

Peimbert, M., Luridiana, V., \& Peimbert, A. 2007, ApJ, 666, 636

Pelupessy, F. I., van der Werf, P. P., \& Icke, V. 2004, A\&A, 422, 55

Pelupessy, F. I., Papadopoulos, P. P., \& van der Werf, P. 2006, ApJ, 645, 1024

Petrosian, V. 1976, ApJ, 209, 1

Pisano, D. J., Koo, D. C., Willmer, C. N. A., Noeske, K. G., \& Phillips, A. C. 2005, ApJ, 630, L25

Poggianti, B. M., Smail, I., Dressler, A., et al. 1997, ApJ, 518, 576

Pustilnik, S. A., Kniazev, A. Y., Lipovetsky, V. A., \& Ugryumov, A. V. 2001, A\&A, 373, 24

Pustilnik, S. A., Kniazev, A. Y., Pramskij, A. G., Ugryumov, A. V., \& Masegosa, J. 2003, A\&A, 409, 917

Pustilnik, S. A., Pramskij, A. G., \& Kniazev, A. Y. 2004a, A\&A, 425, 51

Pustilnik, S., Kniazev, A., Pramskij, A., et al. 2004b, A\&A, 419, 469

Pustilnik, S. A., Kniazev, A. Y., \& Pramskij, A. G. 2005, A\&A, 443, 91

Pustilnik, S. A., Engels, D., Kniazev, A. Y., et al. 2006, AstL, 32, 228

Recchi, S., Matteucci, F., D'Ercole, A., \& Tosi, M. 2002, A\&A, 384, 799

Sargent, W. L. W., \& Searle, L. 1970, ApJ, 162, L155

Schlegel, D. J., Finkbeiner, D. P., \& Davis, M. 1998, ApJ, 500, 525

Schaerer, D., \& de Koter, A. 1997, A\&A, 322, 598

Schaerer, D., \& Vacca, W. D. 1998, ApJ, 497, 618

Schulte-Ladbeck, R. E., Hopp, U., Crone, M. M., \& Greggio, L. 1999, ApJ, 525, 709

Shields, G. A., \& Tinsley, B. M. 1976, ApJ, 203, 66

Takamiya, M. 1999, ApJS, 122, 109

Taylor, C. L., Brinks, E., \& Skillman, E. D. 1993, AJ, 105, 128

Telles, E., Melnick, J., \& Terlevich, R. 1997, MNRAS, 288, 78

Terlevich, R., Melnick, J., Masegosa, J., Moles, M., \& Copetti, M. V. F. 1991, A\&AS, 91, 285

Thuan, T. X. 1991, in Massive Stars in Starbursts, ed. C. Leitherer et al. (Cambridge University Press), 183

Thuan, T. X., \& Martin, G. E. 1981, ApJ, 247, 823

Thuan, T. X., \& Izotov, Y. I. 2005, ApJS, 161, 240

Thuan, T. X., Izotov, Y. I., \& Lipovetsky, V. A. 1995, ApJ, 445, 108

Thuan, T. X., Izotov, Y. I., \& Lipovetsky, V. A. 1997, ApJ, 477, 661

Thuan, T. X., Izotov, Y. I., \& Foltz, C. B. 1999, ApJ, 525, 105

Thuan, T. X., Hibbard, J. E., \& Lévrier, F. 2004, ApJ, 128, 617

Tosi, M., Sabbi, E., Bellazzini, M., et al. 2001, AJ, 122, 1271

van Zee, L. 2000, AJ, 119, 2757

Vanzi, L., Hunt, L. K., Thuan, T. X., \& Izotov, Y. I. 2000, A\&A, 363, 493

Vigroux, L., Thuan, T. X., Vader, J. P., \& Lachièze-Rey, M. 1986, AJ, 91, 70

Vilchez, J. M. 1995, AJ, 110, 1090

Whitford, A. E. 1958, AJ, 63, 201

Youngblood, A. J., \& Hunter, D. A. 1999, ApJ, 519, 55 